\title{
The Proceedings of the International Conference
}

on Creationism

Volume 8

Print Reference: Pages 133-151

Article 7

2018

\section{An Overview of the Independent Histories of the Human $Y$ Chromosome and the Human Mitochondrial chromosome}

Robert W. Carter

Stephen Lee

University of Idaho

John C. Sanford

Cornell University, Cornell University College of Agriculture and Life Sciences School of Integrative Plant

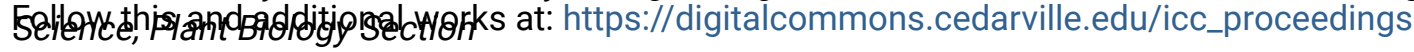

DigitalCommons@Cedarville provides a publication platform for fully open access journals, which means that all articles are available on the Internet to all users immediately upon publication. However, the opinions and sentiments expressed by the authors of articles published in our journals do not necessarily indicate the endorsement or reflect the views of DigitalCommons@Cedarville, the Centennial Library, or Cedarville University and its employees. The authors are solely responsible for the content of their work. Please address questions to dc@cedarville.edu.

Browse the contents of this volume of The Proceedings of the International Conference on Creationism.

\section{Recommended Citation}

Carter, R.W., S.S. Lee, and J.C. Sanford. An overview of the independent histories of the human Ychromosome and the human mitochondrial chromosome. 2018. In Proceedings of the Eighth International Conference on Creationism, ed. J.H. Whitmore, pp. 133-151. Pittsburgh, Pennsylvania: Creation Science Fellowship. 


\title{
AN OVERVIEW OF THE INDEPENDENT HISTORIES OF THE HUMAN Y CHROMOSOME AND THE HUMAN MITOCHONDRIAL CHROMOSOME
}

\author{
Robert W. Carter, FMS Foundation, 877 Marshall Rd, Waterloo, NY 13165, USA, rcarter@FMSFound.org \\ Stephen S. Lee, Statistical Science Department, University of Idaho, Moscow, ID 83843, USA, stevel@uidaho.edu \\ John C. Sanford, FMS Foundation, 877 Marshall Rd, Waterloo, NY 13165, USA, rcarter@FMSFound.org
} ABSTRACT

The existence of a literal Adam and Eve is hotly debated, even within the Christian body. Now that many full-length human $\mathrm{Y}(\mathrm{chrY})$ and mitochondrial (chrM) chromosome sequences have been sequenced and made publicly available, it may be possible to bring clarity to this question. We have used these data to comprehensively analyze the historical changes in these two chromosomes, starting with the sequences of people alive today, and working backwards to the ancestral sequence of the family groups to which they belong. The analyses of the chrY and chrM histories were done separately and in parallel. Remarkably, both analyses gave very similar results. First, the pattern displayed in both datasets supports a massive expansion of the human lineage, with multiple new branches forming from closelyrelated individuals. Second, for both chromosomes, the mutation rate along each branch has not been the same through time. Third, both phylogenetic trees display a starburst pattern that centers around specific historical individuals, nearly all of whom lived in the Middle East. Fourth, we can know with a very high degree of confidence the actual sequences of the historical individuals that gave rise to each branch in both family trees. Fifth, within a reasonable margin of error we can approximate the sequence of Y chromosome Adam/Noah and Mitochondrial Eve. Sixth, given a few reasonable assumptions, we can estimate the time to Y Chromosome Adam/Noah and Mitochondrial Eve. Both individuals lived less than 10,000 years ago, which is most consistent with a biblical timeframe. Lastly, recurrent mutations are extremely common, and many of them are associated with epigenetic $\mathrm{CpG}$ sites, meaning mutation accumulation is not free of environmental influence and many mutations may have accumulated in different lineages in parallel. The genetic evidence strongly suggests that Y Chromosome Adam/Noah and Mitochondrial Eve were not just real people, they were the progenitors of us all. In this light, there is every reason to believe that they were the Adam/Noah and Eve of the Bible.

\section{KEY WORDS}

Adam, Eve, genetics, mutation, Y chromosome, mitochondria, ancestral reconstruction, molecular clock

\section{INTRODUCTION}

The Bible describes the creation of a first founding couple of all humanity, Adam and Eve. Even though the early church readily accepted the reality of Adam and Eve, this has been a highly controversial subject for the past several centuries, specifically after the rise of Darwinism. One of the more controversial aspects of this debate deals with the evidence pointing back to a single man ("Y chromosome Adam", c.f. Karafet et al. 2008) and a single woman ("mitochondrial Eve", c.f. Cann et al. 1987) who supposedly lived one to two hundred thousand years ago, much further back than the biblical timescale allows. In this paper, we will analyze these claims and attempt to show that 1) Adam and Eve are a concrete reality, 2) the timeframe in which they lived is much more recent than evolutionary calculations suggest, and 3) variable branch lengths on the $Y$ and mitochondrial family tree strongly indicate that mutations have not accumulated at the same rate across time or geography. This last point is a direct challenge to the "molecular clock hypothesis" that is, in turn, behind all evolutionary speculations about the timing of genetic events, including the "Out-of-Africa" dispersion and the time when Y chromosome Adam and mitochondrial Eve lived.

Rapid advances in DNA sequencing have led to an enormous wealth of genomic data, including "whole-chromosome" databases such as the 1000 Genomes Project (2010) and the Simons Genome Diversity Project (Malik et al. 2016). These new data have opened an unprecedented window into human genetic history. For example, a recently published study of over 13,000 Y chromosome single nucleotide variations (SNVs) by Hallast et al. (2015) revealed various previously-hidden aspects of worldwide $\mathrm{Y}$ chromosome diversity. This was quickly followed by an analysis by Poznik et al. (2016) that included more than 60,000 SNVs, 1,400 indels, 110 copy-number variations, and 3,200 short tandem repeats from more than 1,200 full-length chromosomes sequenced by the 1000 Genomes Project. These new studies provide important new insights into human genetic history, and the discovery process has just begun.

The Y and mitochondrial chromosomes can be subdivided into distinct 'haplogroups' by the particular set of mutations each carries. Due to technological limitations, haplogroup identification was traditionally limited to a small set of specific discriminating alleles. For example, the first successful typing method for mitochondria involved restriction endonuclease digestion (Johnson et al. 1983). This allowed for the identification of the major clades (after much work and many revisions). Later, sequencing of the two hypervariable regions (HVRI and HVRII) allowed for improved 
clade resolution (Handt et al. 1998). Y chromosome typing began with more limited sampling of short tandem repeats (STRs) (Purps et al. 2014) and Alu insertion events (Romualdi et al. 2002). Several decades ago, geneticists moved into analyses on the level of single nucleotide variations (SNVs). It was not until recently that nearly complete whole-chromosome SNV data became available for the Y chromosome. Now that nearly full-length sequences are abundant and readily accessible (Smith 2015), haplogroup identification is no longer limited to just a limited set of specific alleles but can employ all variation data found within representative members of all known clades. This is powerful information that can help answer crucial questions regarding human origins.

Using several different methods, researchers can create phylogenetic trees that reflect the genetic history of any given set of related people living today. The tree-building algorithms are forced to use approximations when comparing sequence data, and thus the nodes and interior branches do not necessarily reflect real individuals that lived in the past. However, as we will demonstrate, in the case of the human $\mathrm{Y}$ and mitochondrial gene trees, each branch point on each tree reflects a historical individual that passed one or more de novo mutations to a child. This means that any branch arises at a specific time, in a specific individual, and that event provides an informative reference point that enables the study of both the group founder and his or her descendants.

After combining related sequences into natural haplogroups, it is possible to reconstruct the ancestral sequence of each group. This can be done with a high degree of confidence. Ancestral sequence reconstruction dates back as far as the pioneering work of Pauling and Zuckerkandl (1963) who introduced the term 'paleogenetics' in the early 1960s. This field has a strong mathematical basis that has continuously advanced over the decades since work was begun. Early parsimony methods like those of Jermann et al. (1995) were largely eclipsed by maximum likelihood methods like those of Pupko et al. (2000), which were followed Bayesian methods like those of Huelsenbeck and Bollback (2001).

Historical sequence reconstructions have many complexities and are subject to multiple confounding factors, such as the presence of incomplete lineage sorting, genomic rearrangements, gene duplication and deletion, varying mutation rates over time, gene conversion, and differing rates of specific mutations. Worse, phylogenetic reconstructions will always yield a tree, even for unrelated organisms (i.e., different created kinds). Furthermore, the assumption that an accurate 'molecular clock' exists can also affect the final shape of the tree. Yet the presence of an accurate molecular clock is a highly-debatable subject (Wood 2012, 2013; Tomkins and Bergman 2015; Jeanson 2016). Despite the controversy, the molecular clock hypothesis has a profound effect on how phylogenetic trees are constructed. For example, the most dissimilar sequences are usually labeled as the oldest, and are generally shown as outlying branches, ignoring the possibility that they might be the same age as the others, but having more mutations.

We understand these complexities, but for special chromosomes such as chrY and chrM (i.e., for non-recombining DNA elements with uniparental inheritance), the reconstruction of the ancestral sequence can be relatively simple. There is often no need to identify regions of synteny among diverse lineages, for example, and the alignment is often trivial. This gives us the unprecedented opportunity to examine, in parallel, the histories of both chromosomes. This has allowed us to shed new light on the genetics of both our primary patriarchal ancestor and our primary matriarchal ancestor.

\section{Methods}

The latest $Y$ chromosome, mitochondrial (see Diroma et al. 2014), and chromosome 22 sequence data were obtained from the 1000 Genomes Project page (accessed 17 Apr 2015). High-coverage, high-quality, long-read Y chromosome data for 25 of the 1000 Genomes individuals was obtained from Complete Genomics (ftp://ftp2.completegenomics.com/Multigenome_summaries/ Complete_Public_Genomes_69genomes_VQHIGH_testvariants. tsv, accessed 3 Feb 2015). High-coverage Y chromosome data for 176 additional individuals from a diverse worldwide sampling was obtained in the Simons Genome Diversity Project (Malik et al. 2016). We constructed a full distance matrix for the 1000 Genomes $\mathrm{Y}$ and mitochondrial sequence data and then created naive neighbor-joining trees using MEGA, version 7 (Tamura et al. 2013)(Figs 1-3).

Since our methodology requires multiple sequences within each group under consideration, two Y chromosome sequences (HG03742 and $\mathrm{HG} 02040$, from haplogroups $\mathrm{K} 2 \mathrm{a} 1 *$ and $\mathrm{F}^{*}$, respectively) were dropped from the analysis. The International Society for Genetic Genealogy (ISOGG) has curated a detailed table of Y chromosome variants (isogg.org/tree/ISOGG_YDNATreeTrunk. html, accessed 8 Feb 2016). We consulted this to double check the 1000 Genomes haplotype assignments and were surprised that two of the "A1b" sequences were strongly associated with variants that define haplogroup A0. Since the generally accepted phylogenetic root falls between these two clades, we split them into groups A0 and A1, following Karmin et al. (2015).

Y chromosome haplogroup A0 and mitochondrial haplogroup L0 were used as outgroups. We filtered out any location where more than half of the readings were missing data or where missing data created a complex situation where the called ancestral allele was incongruent to the main phylogeny.

We reconstructed the ancestral sequence for each major haplogroup using a simple decision tree similar to that of Pauling and Zuckerkandl (1963). In order to assign ancestral alleles, the state of that allele within a group is compared to its state outside the group. There are four possible results:

A. No within-group variability and all other groups fixed for the alternate allele. The change must have happened within the ancestral stem of the group. It is unreasonable to think that multiple parallel mutations happened in all groups but the one under consideration, so this can be discounted. In these cases, the ancestral allele is set to the "Out" value. A special case arises when considering the outgroup (either included by design or by default as the deepestbranching group on an unrooted tree). If the outgroup is different from all others, it is impossible to directly identify the ancestral state, for the mutation could have happened on either side of the main stem. That is, along the branch that leads to the outgroup or 


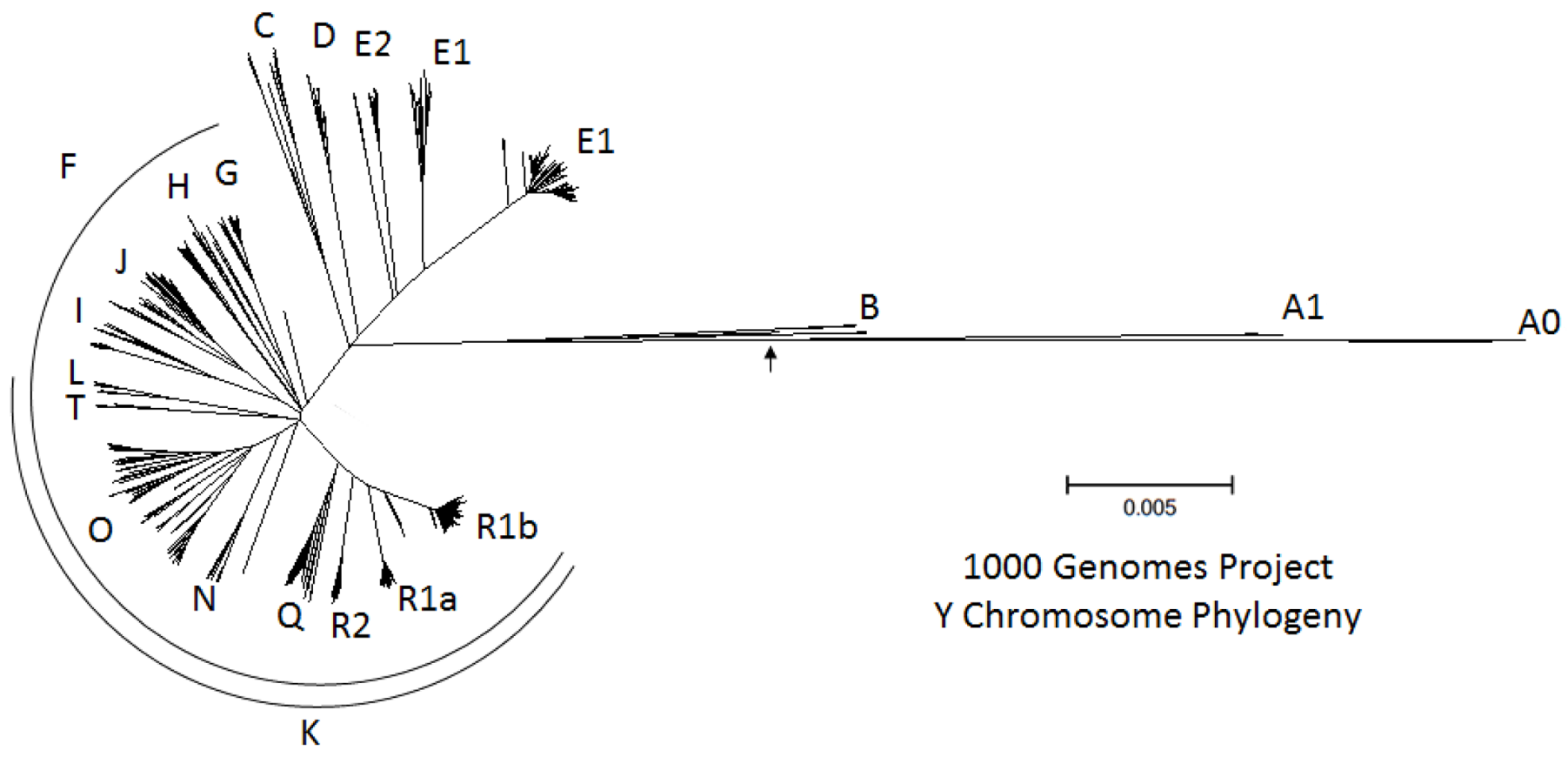

Figure 1. An unrooted neighbor-joining phylogenetic tree of 1,233 Y chromosomes from the 1000 Genome Project. Semi-circles denote commonly-used macrohaplogroup names. The small arrow denotes the approximate position of the evolutionary root. The scale bar represents approximately 300 mutations.

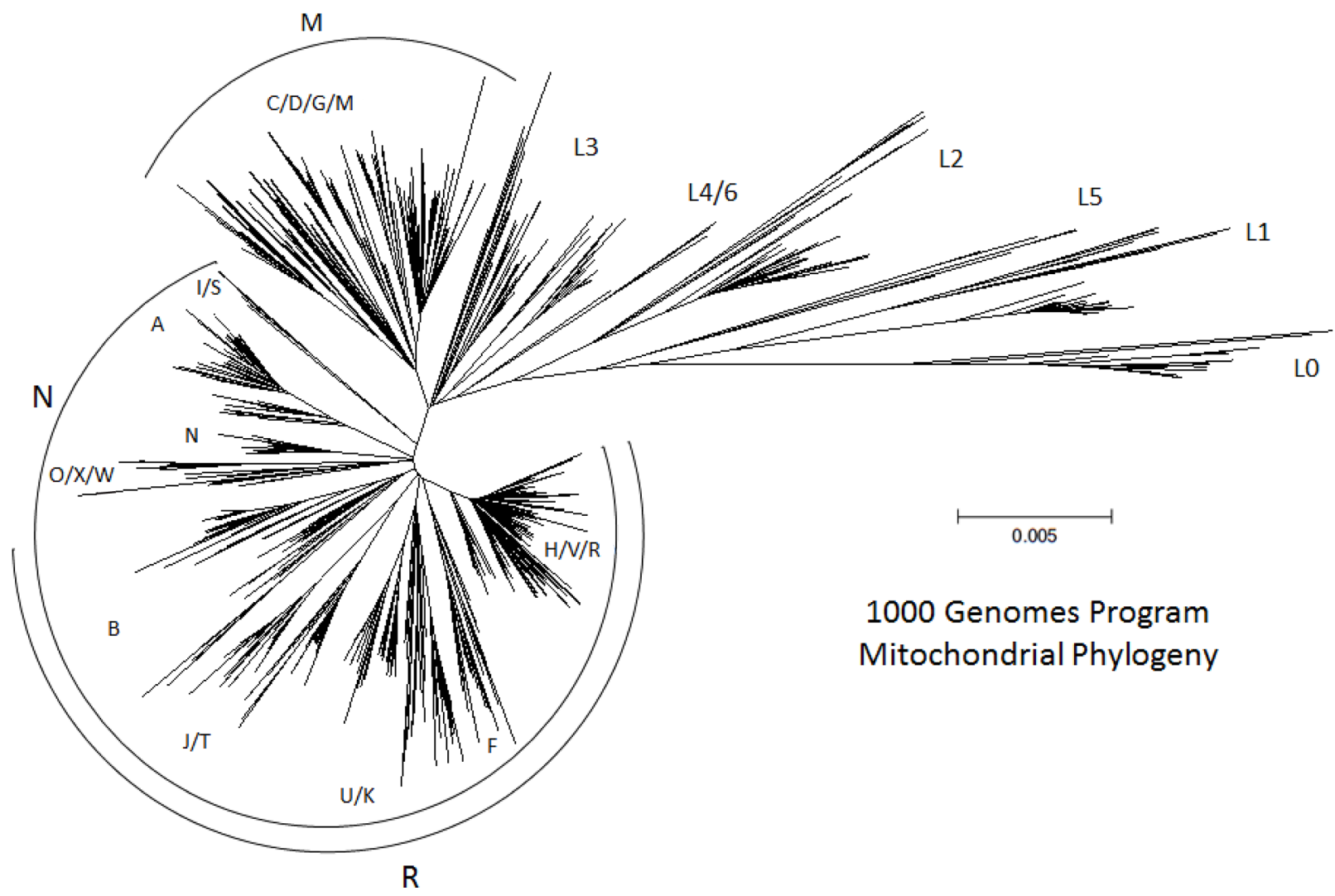

Figure 2. An unrooted neighbor-joining phylogenetic tree of the mitochondrial chromosomes from the 1000 Genome Project. Details are similar to those of Fig. 1, with the addition of Australia/Pacific Islands. The scale bar represents approximately 14 mutations. 


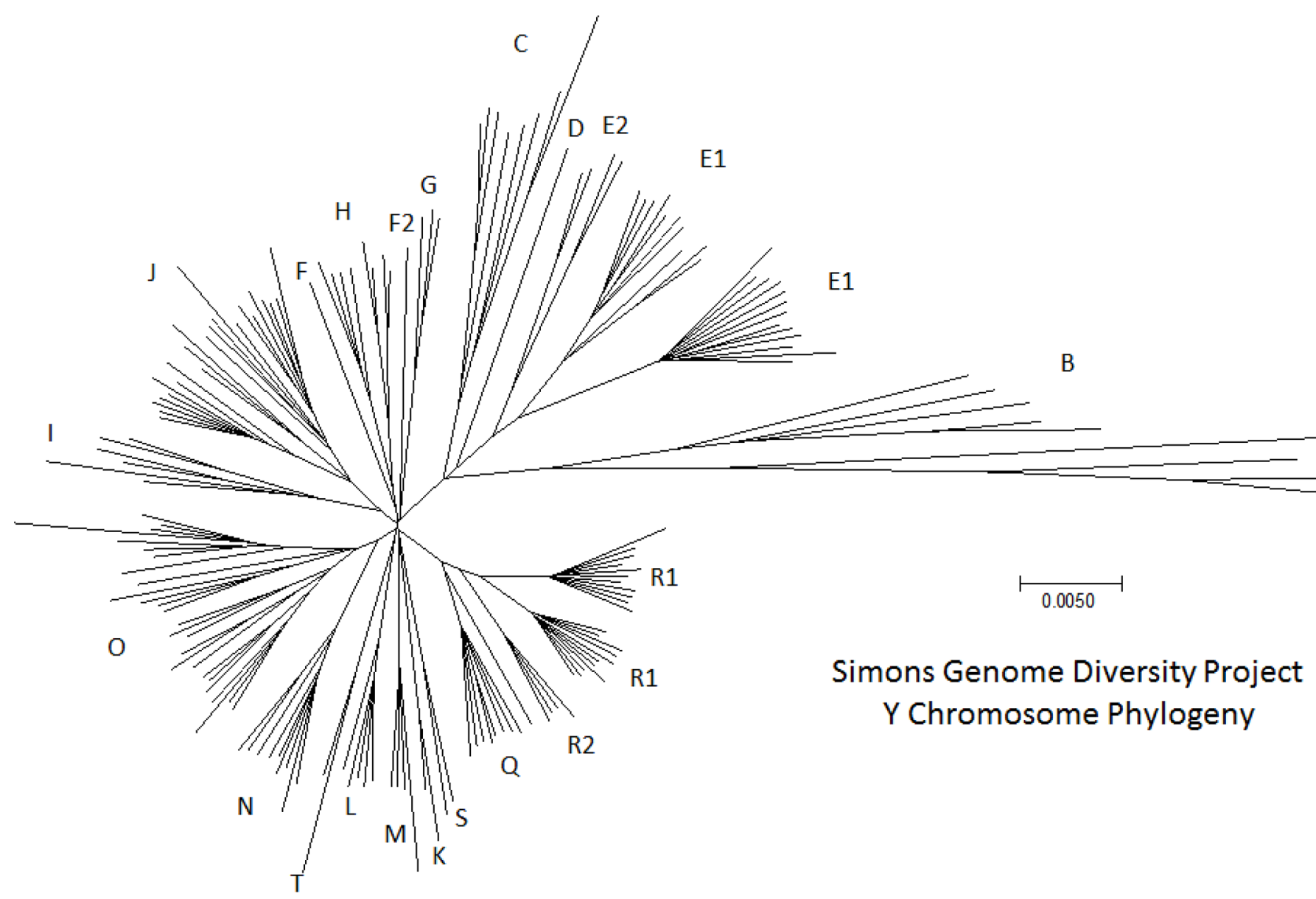

Figure 3. An unrooted neighbor-joining phylogenetic tree of the Y chromosomes from the Simons Genome Diversity Project. Unlike 1000 Genomes, which sampled heavily from specific populations, SGDP attempted to sample from a much wider range of peoples. The result is a tree that better represents total worldwide Y chromosome diversity. Noah and/or Shem, Ham, and Japheth would be located near the center of the starburst. The scale bar represents approximately 700 mutations.

along the branch that leads to the rest. "Using prior knowledge," Poznik et al. (2016) chose the midpoint between A0 and A1 as the Y root. We deliberately chose to not do this. Thus, the outgroup we used (A0) was not used in any further analyses.

B. Within-group variability and all other groups are fixed for either allele. This is really a special case of (A) and increases the likelihood of accurate reconstruction. For every level of branching in the tree, the probability that only one sub-branch contains the original equals the probability that the mutation happened in all other branches, which rapidly becomes vanishingly small. Thus, the more rare the variant, the more likely an accurate call.

C. No within-group variability and the outgroup is variable. This is, of course, the reverse of (B). In this case, the ancestral allele is most likely the one fixed in the group, but it depends on the level of branching at which the allele is also found in other groups. Reversions are possible (frequent, in fact, at specific locations in both chromosomes). The more common the allele, and the more branches in which it is found, the more likely it is the original. In these cases, the ancestral allele is set to the "In" value.

D. Within-group variability and other groups are also variable. This case requires special tests. Many such situations are due to 'private' mutations that occur in only one member of a specific group, and can thus be discounted. These could either be due to repeat mutations or sequencing errors, but either way the probability that the rare group allele is the ancestral allele is small. In an infinite alleles model, this should not be possible. But sequencing errors and the occasional homoplasy do create them, and they have been noted previously (Hallast et al. 2015). Second-pass tests were performed in these cases: first, within-group private mutations were removed and the samples were rerun. If the conflict was not resolved, we considered the ancestral state call for the other groups. If no more than three groups were problematic, and if all other groups unanimously called the same ancestral allele, the ancestral allele in the ambiguous cases were set to "Out" allele. For the few remaining cases, if the majority allele was identical in all groups (both the groups with no variability and the ones with variability), the ancestral allele was set to the majority allele.

Poznik et al. (2016) mentioned that pooling sequences into subtrees first (essentially our method) is computationally more efficient and creates a method less prone to difficulties due to homoplasy. It also leads to simple ancestral state reconstruction. Further, our methods allowed for sorting and visual examination of the data at multiple stages. This allowed for double-checking and validation of multiple conclusions we drew from the analyses that would not have been possible using an off-the-shelf phylogeny package. All 
phylogenetic methods (including parsimony, maximum likelihood, and Bayesian approaches) yield slightly different results (HansonSmith et al. 2010; Groussin et al. 2015). Thus, it is expected that our ancestral reconstruction methods will produce results slightly different from other methods. Yet, as long as all sequences are being compared to the correct ancestor, as long as that ancestor is not biased in the direction of some descendant sequences over others (as would occur in a strict consensus model, for example), and as long as enough mutations have occurred in each lineage to produce a statistically-robust average, ancestral reconstruction should produce accurate results.

The distances of each sequence to its group ancestor was calculated and group averages and standard deviations were tabulated. Sequence manipulation and most calculations were performed in Perl or MEGA. We used Tukey's Multiple Comparisons of Means to calculate family-wise $95 \%$ confidence intervals for all pair-wise divergence differences among all sequences compared to each ancestral sequence.

Once we saw that the rates of mutation accumulation were not identical in all lineages, we decided to explore why this might be true. We obtained the sequence data from the 50,000th-generation of E. coli (Tenaillon et al. 2016) grown in the Long-Term Evolution Experiment (LTEE) pioneered by Richard Lenski. We compared the relative proportion of each of the $12 \mathrm{SNV}$ types in the bacterial chromosomes and within human chromosome 22.

\section{RESULTS}

1. A comprehensive phylogeny for the $Y$ and mitochondrial chromosomes: The unrooted neighbor-joining phylogenic trees for the $\mathrm{Y}$ and mitochondrial chromosomes are show in (Figs. 1-3). There are several interesting things that can be seen in these images. First, there is always a clear, central starburst pattern. Since most new mutations are lost to drift with time (Rupe and Sanford 2013), the only way to capture a pattern like this is if the human population expanded extremely rapidly and/or if it had an exceptionally high mutation rate at an earlier period of its history. Comparing the natural groupings revealed in the phylogenetic trees to the nearestneighbor data allowed us to identity 11 major haplotypes for chrY and 16 major haplogroups for chrM. Some of these were collapsed into larger groups when ancestral reconstruction revealed that they had identical ancestors.

2. Ancestral sequences for each $Y$ chromosome haplogroup: Applying the first-pass tests (cases A, B, and C in the ancestral reconstruction methods described above) led to unambiguous ancestral predictions for $98.3 \%$ of all variable positions among all $\mathrm{Y}$ chromosome haplogroups. This is similar to the reported ambiguity found in the mitochondrial dataset of Carter et al. (2008). The data were complicated by the presence of multiple apparent homoplasies. These are mutations that occur in parallel in independent lineages, including hundreds of locations in the mitochondrial data and thousands of locations in the Y chromosome data. Nearly all were resolved using the second-pass test (the special cases mentioned in Methods). Especially important was the removal of unique withingroup alleles (i.e., the only reason the homoplasy existed was that an allele associated with a major phylogenetic branch point also appeared in a single individual in an unrelated group). This either revealed many sequencing errors, which is unlikely, or thousands of examples of repeating mutations or gene conversion events at the same locations in disconnected lineages, which has significant implications for phylogenetics.

Sorting and visual examination of the 1000 Genomes data showed that there were no ancestral allele calls that contradicted the main branches on the standard Y chromosome phylogenetic tree (c.f. Scozzari et al. 2014). However, several ancestral mitochondrial allele calls were different from the most recent phylogenetic work (e.g., Behar et al. 2012). There was so much recurrent mutation at several places that the ancestral allele was uncertain: either there was not a clear consensus, homoplasy existed in the majority of branches, or the pattern made no sense compared to the overall tree. Thus, contrary to Behar et al. (2012), we found no differences in the ancestral sequence of haplogroups L4 and L6, and haplogroup $\mathrm{I} / \mathrm{S}$ was removed from macrohaplogroup $\mathrm{N}$ by a single mutation (at position 10398), whereas all of the other macrohaplogroup $\mathrm{R}$ branches split off directly from a common node after that.

3. Differences among haplogroup founders: The distance matrix for the Y chromosome haplogroup founders is given in Table 1. It reveals three large clusters of closely-related $Y$ chromosome groups. The distance matrix for the mitochondrial haplogroup founders is given in Table 2. It shows that multiple major lineages (e.g., B, $\mathrm{F}, \mathrm{H} / \mathrm{V} / \mathrm{R}, \mathrm{J} / \mathrm{T}$, and $\mathrm{U} / \mathrm{K}$ ) branch off directly and simultaneously (from a tree-building perspective) from a single ancestral sequence (in this case, the founder of macrohaplogroup R). The presence of multiple early women who were both closely related and who were the founders of large proportions of the current world population is surprising, to say the least, unless one is considering biblical history. If all haplogroups branched off from within a population of $\sim 10,000$ individuals, founders should essentially never be closely related. It is also important to note that the ancestor of the "Out of Africa" clade (L3) is identical to the ancestor of macrohaplogroup M. There was no discernable time between the rise of the group

Table 1. Distances between all Y chromosome haplogroup ancestors. There are several major groupings evident in this table (shaded areas), including a group that includes the closely-related ancestors of $\mathrm{L} / \mathrm{T}, \mathrm{N} / \mathrm{O}$, and $\mathrm{Q} / \mathrm{R}$ (macrohaplogroup $\mathrm{K}$ ), as well as the ancestors of $\mathrm{G}, \mathrm{H}$ and $\mathrm{I} / \mathrm{J}$, and the ancestors of $\mathrm{D} / \mathrm{E}$ and $\mathrm{C}$. These three groups represent most of the $\mathrm{Y}$ chromosome lineages in the world. Actually, macrohaplogroup $\mathrm{K}$ has that distinction by itself, but the other groups still represent a significant percentage of world ancestry. Thus, the majority of worldwide Y chromosome haplogroups immediately descend from one of three macrohaplogroup ancestors.

\begin{tabular}{|c|c|c|c|c|c|c|c|c|c|c|}
\hline & $\mathrm{A} 1$ & $\mathrm{~B}$ & $\mathrm{C}$ & $\mathrm{D} / \mathrm{E}$ & $\mathrm{G}$ & $\mathrm{H}$ & $\mathrm{I} / \mathrm{J}$ & $\mathrm{L} / \mathrm{T}$ & $\mathrm{N} / \mathrm{O}$ & $\mathrm{Q} / \mathrm{R}$ \\
\hline $\mathrm{A} 1$ & & 422 & 653 & 649 & 816 & 817 & 824 & 840 & 841 & 841 \\
\hline $\mathrm{B}$ & 422 & & 233 & 229 & 396 & 397 & 404 & 420 & 421 & 421 \\
\hline $\mathrm{C}$ & 653 & 233 & & 4 & 163 & 164 & 171 & 187 & 188 & 188 \\
\hline $\mathrm{D} / \mathrm{E}$ & 649 & 229 & 4 & & 167 & 168 & 175 & 191 & 192 & 192 \\
\hline $\mathrm{G}$ & 816 & 396 & 163 & 167 & & 1 & 8 & 24 & 25 & 25 \\
\hline $\mathrm{H}$ & 817 & 397 & 164 & 168 & 1 & & 7 & 23 & 24 & 24 \\
\hline $\mathrm{I} / \mathrm{J}$ & 824 & 404 & 171 & 175 & 8 & 7 & & 16 & 17 & 17 \\
\hline $\mathrm{L} / \mathrm{T}$ & 840 & 420 & 187 & 191 & 24 & 23 & 16 & & 1 & 1 \\
\hline $\mathrm{N} / \mathrm{O}$ & 841 & 421 & 188 & 192 & 25 & 24 & 17 & 1 & & 0 \\
\hline $\mathrm{Q} / \mathrm{R}$ & 841 & 421 & 188 & 192 & 25 & 24 & 17 & 1 & 0 & \\
\hline
\end{tabular}


Table 2. Distances between all mitochondrial chromosome haplogroup ancestors. There are fewer groups here than in Fig. 2 because we combined the members of macrohaplogroup $\mathrm{R}$ (they were found to have identical mitochondrial ancestors). Only a small number of mutations accumulated in the human population prior to our spreading out across the world.

\begin{tabular}{|c|c|c|c|c|c|c|c|c|c|}
\hline & L1 & L5 & L2 & L4/6 & L3 & M & I/S & N & R \\
\hline L1 & & 5 & 14 & 18 & 20 & 20 & 22 & 23 & 25 \\
\hline L5 & 5 & & 9 & 13 & 15 & 15 & 17 & 18 & 20 \\
\hline L2 & 14 & 9 & & 4 & 6 & 6 & 10 & 11 & 13 \\
\hline L4/6 & 18 & 13 & 4 & & 2 & 2 & 6 & 7 & 9 \\
\hline L3 & 20 & 15 & 6 & 2 & & 0 & 4 & 5 & 7 \\
\hline M & 20 & 15 & 6 & 2 & 0 & & 4 & 5 & 7 \\
\hline I/S & 22 & 17 & 10 & 6 & 4 & 4 & & 1 & 3 \\
\hline N & 23 & 18 & 11 & 7 & 5 & 5 & 1 & & 2 \\
\hline R & 25 & 20 & 13 & 9 & 7 & 7 & 3 & 2 & \\
\hline
\end{tabular}

that supposedly remained in Africa and the group that supposedly left. That is unexpected under the Out of Africa Model.

4. Phylogenetic relationships between haplogroup founders: Both phylogenies have an asymmetrical, star-like topology. Most of the major haplogroup founders are tightly clustered, suggesting a small initial population that underwent explosive population growth. This is not surprising, as genomic data support this idea in general, but from the chrY data it is clear that most men in the world are descended from a small number of closely-related individuals. A similar thing can be said of the mitochondrial lineages. Since mutation occurs more or less at random, and since the mutation rate in the $\mathrm{Y}$ and mitochondrial chromosomes can be less than one per chromosome per generation, were we to run the clock backward and start the post-Flood dispersion again, we would not necessarily get the exact same tree. However, one would get a similar pattern. The asymmetry in both trees is interesting in that is it so similar. A long branch separates the Eurasian groups from groups more closely-associated with Africa, and then rare African groups form long, spidery branches from that point. Clearly, ancient demographic processes are shaping the genetic landscape, but how much of this is demography and how much of this reflects the preand immediately post-Babel population genetics is unknown.

5. The effects of low coverage: The main difficulty with the divergence data comes from the fact that the 1000 Genomes data are low coverage. Low sequence coverage makes it difficult to detect short indels and copy number variations, but any effect is expected to be small since, with only a few exceptions, the types of variation known to exist cover only a limited number of SNVs.
And even though a certain number of low frequency variants were expected to be missed, when we compared divergence to the sequence coverage, no trend towards higher divergence with lower coverage was revealed (data not shown).

6. Validation using high-coverage sequencing data: We repeated our methods on the Y chromosomes of the 25 individuals included in the Complete Genomics panel of 69 high-coverage genomes. We built a phylogenetic tree and then did extensive comparisons between the Complete Genomics, 1000 Genomes, and Poznik et al. (2016) data. Among these 25 Y chromosomes, 1000 Genomes detected 4,689 SNVs. Complete Genomics added another 377 in those same regions and an additional 5,010 outside the areas covered by 1000 Genomes (Fig. 4).

If we only consider those variable positions detected by both 1000 Genomes and Complete Genomics, there are only 39 points (out of 25 x 4,689 readings, or $0.034 \%$ ), distributed among 23 genomic locations, where the two data sets contradicted one another. If we assume that Complete Genomics always corrects 1000 Genomes, in about half the cases (12/23) Complete Genomics calls a private allele that 1000 Genomes missed. This is an average of 0.44 missed private alleles per person. But this rate would be much lower in the larger data set of 1,233 Y chromosomes. Visually examining these 23 locations in the larger data set revealed that 15 of these locations are variable within one of the major haplogroups and another two are fixed within a haplogroup (and thus none of these are private alleles in 1000 Genomes). The remaining six locations are complex, with much homoplasy but always with a clear majority allele. Obviously, we have reached the limits of current sequencing technology, but the expected number of false positives in the 1000 Genomes Y chromosome SNV dataset is less than 1 per person, on average.

Of the 377 places where Complete Genomics called a variable allele that was missed by 1000 Genomes, $75.9 \%$ identified a private allele. This is not surprising when you consider that 1000 Genomes is expected to miss a greater percentage of rare variation due to low average sequence coverage. However, false negatives are still much less than one per person.

7. Divergence of individuals from their haplogroup founder: The time elapsed between the ancestor of any haplogroup and the modern members of that group, by definition, is always the same for all individuals in the group. Fig. 5 shows the average distance from each Y chromosome haplogroup founder to the members of that specific haplogroup. We were initially surprised to observe that

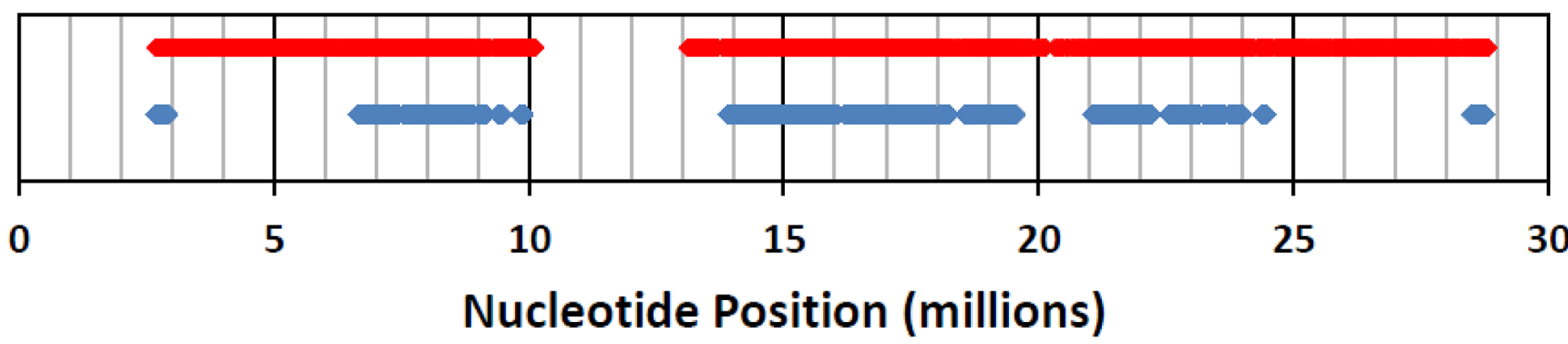

Figure 4. SNV locations in the 1000 Genomes (blue) and Complete Genomics (red) datasets. The Y chromosome centromere starts at about nucleotide position 10.3 million, which accounts for the large gap there. The long heterochromatic area (beyond nucleotide position 30 million) was not sequenced in either study. 
for nearly every haplogroup founder there were modern individuals with sequences who were technically outside of his descendent haplogroup(s), yet were actually closer to the founder than many of his living descendants. After replacing the divergence values by rank order, a stronger picture emerges (Fig. 6). For example, in the evolutionary model, "Ancestor A1" is the ancestor of every man in this study. Yet the three sequences in haplogroup A1 were ranked much higher (i.e., closer) to Ancestor A1 than expected. Since there were so few A1 sequences available, we might discount this observation were it not for further examples. A similar pattern is seen among the sequences in haplogroup $\mathrm{B}$. With the exception of the individuals belonging to haplogroup $\mathrm{A}$, evolutionists believe "Ancestor B" is the ancestor of everyone in this study. Yet, the B individuals were also ranked much higher than expected. And even though the ancestors of haplogroups $\mathrm{C}$ and $\mathrm{D} / \mathrm{E}$ are only four SNVs apart, the sequences in the two groups rank very differently, with the $\mathrm{C}$ sequences consistently ranking lower (i.e., further away) than the $\mathrm{D} / \mathrm{E}$ sequences (some of which are African).

Clearly, mutational divergence rates might not be constant in all lineages. Because of this, we used Tukey's Multiple Comparisons of Means to calculate family-wise $95 \%$ confidence intervals for all pair-wise divergence differences among the sequences within each major haplogroup of chrY and chrM (Figs. 7 and 8). Under evolutionary assumptions, all pairs should be equally diverged from their common ancestor. Instead, what is seen is that many family pairs have different degrees of divergence (i.e., there were many statistically significant differences among the group pairs).

For example, members of $\mathrm{Y}$ chromosome haplogroups $\mathrm{A} 1$ and $\mathrm{B}$ were significantly less diverged (i.e., picked up fewer mutations in the same amount of time) from Ancestor A1 than members of the other haplogroups. At the same time, haplogroup $\mathrm{C}$ was significantly more diverged from Ancestor $\mathrm{C}$ than the other descendant haplogroups. The differences between the other groups were smaller, but N/O was significantly more diverged and $Q / R$ was significantly less diverged from Ancestor A1 than all others. Regardless of which common ancestor is used for comparison, we get differential divergence rates among the descendant sequence groups.

Among the mitochondrial haplogroups, the one group that stands out is haplogroup L3, which is significantly closer to the L0 ancestor than all other groups except L4/6. Why did the members of this haplogroup accumulate less mutations in the same amount

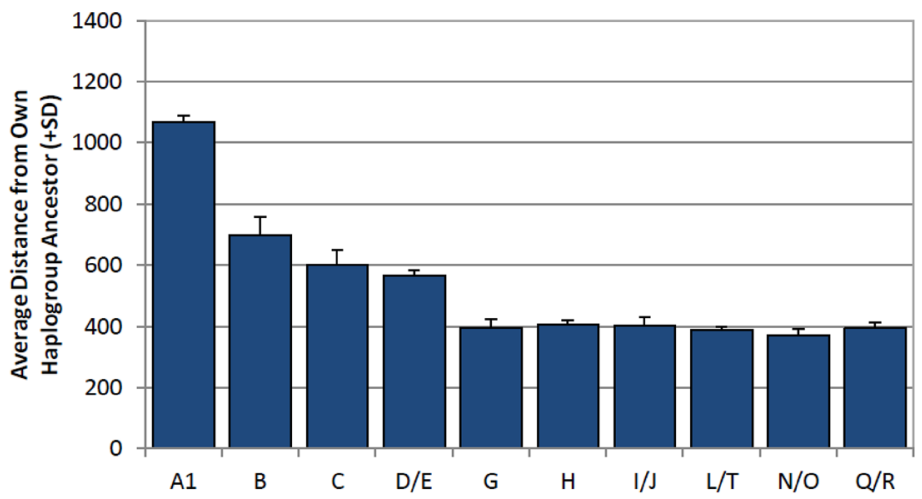

Figure 5. The average distance from each chrY haplogroup ancestor to the members of that haplogroup. Error bars are +1 SD. of evolutionary time? The situation is even more profound if Eve is placed at the L3/M root. Patterns like this exist at all scales. Haplogroup H/V/R displays shorter branch lengths than the related groups $\mathrm{F}$ and $\mathrm{U} / \mathrm{K}$ (see Fig. 1), for example, but they were lumped into the macrohaplogroup $\mathrm{R}$ for these calculations.

The distances (in standard deviations) of all sequences to their haplogroup founder for chrY and chrM are shown as a histogram in Figs. 9 and 10, respectively. Here we see that some sequences are simply more diverged than expected. As we showed above, this is not due to a high false-positive error rate or missing data. As can be seen in the scatter in Figs. 1 and 2, the 'clock' does not tick at the same rate in the family lines of every individual.

Another way to assess the spectrum of accumulating mutations is by generating a histogram of the number of private mutations, meaning mutations that only appear in a single $\mathrm{Y}$ chromosome sequence in the database (Fig. 11). While the status of a private mutation is very much dependent on how closely related other sequences are, this can still give us a rough guess of the allele frequency distribution. Parallel to this, the minor allele frequency plot of Fig. 12 shows that nearly all variants are rare. This is very similar to the mitochondrial data we presented in Carter et al. (2008). The majority of variants between 0.04 and 0.50 are due to structured sampling. That is, if a variant appears along a branch that leads to a major haplogroup, it will appear in all members of that haplogroup. Thus, the three A1 sequences contribute many of the alleles in the $<0.01$ category and the $\mathrm{Q} / \mathrm{R}$ individuals contribute many of the 116 alleles in the $0.30-<0.31$ category.

8. Improved resolution of polytomies and near-polytomies: A polytomy is a point in a phylogeny where more than two branches arise simultaneously. Under most scenarios, all branches are expected to resolve to dichotomies. Since most new mutations are lost to drift over time, the rise of even a single new branch is uncommon. Thus, it should be exceedingly rare for an individual

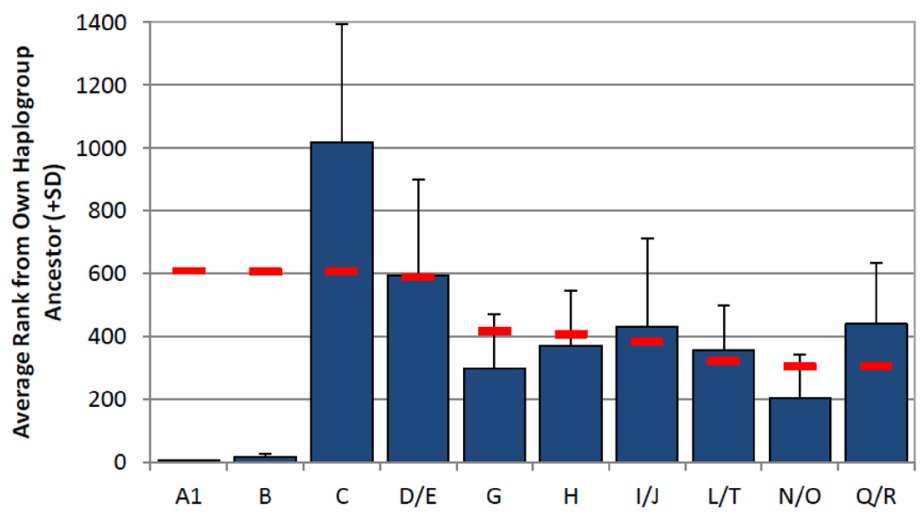

Figure 6. The average rank of the sequences within each chrY haplogroup to their haplogroup ancestor. Error bars are $+1 \mathrm{SD}$. The red bars indicate the expected average rank based on the number of sequences descended from each ancestor, assuming the evolutionary order. Under the molecular clock hypothesis, if all individuals in this database descend from the ancestral A1 node, the A1 sequences should be randomly distributed among the divergence measurements and have an average rank distribution of approximately 615. Instead, the A1 sequences are among the closest sequences. The same is true for the haplogroup B sequences (after excluding A1). After excluding A1 and B, sequences from haplogroup C are more diverged than expected, even if we lumped them with the closelyrelated haplogroup $\mathrm{D} / \mathrm{E}$ sequences. The rest follow independent trends. 
Carter et al. 4 Y Chromosome Noah and mitochondrial Eve 2018 ICC

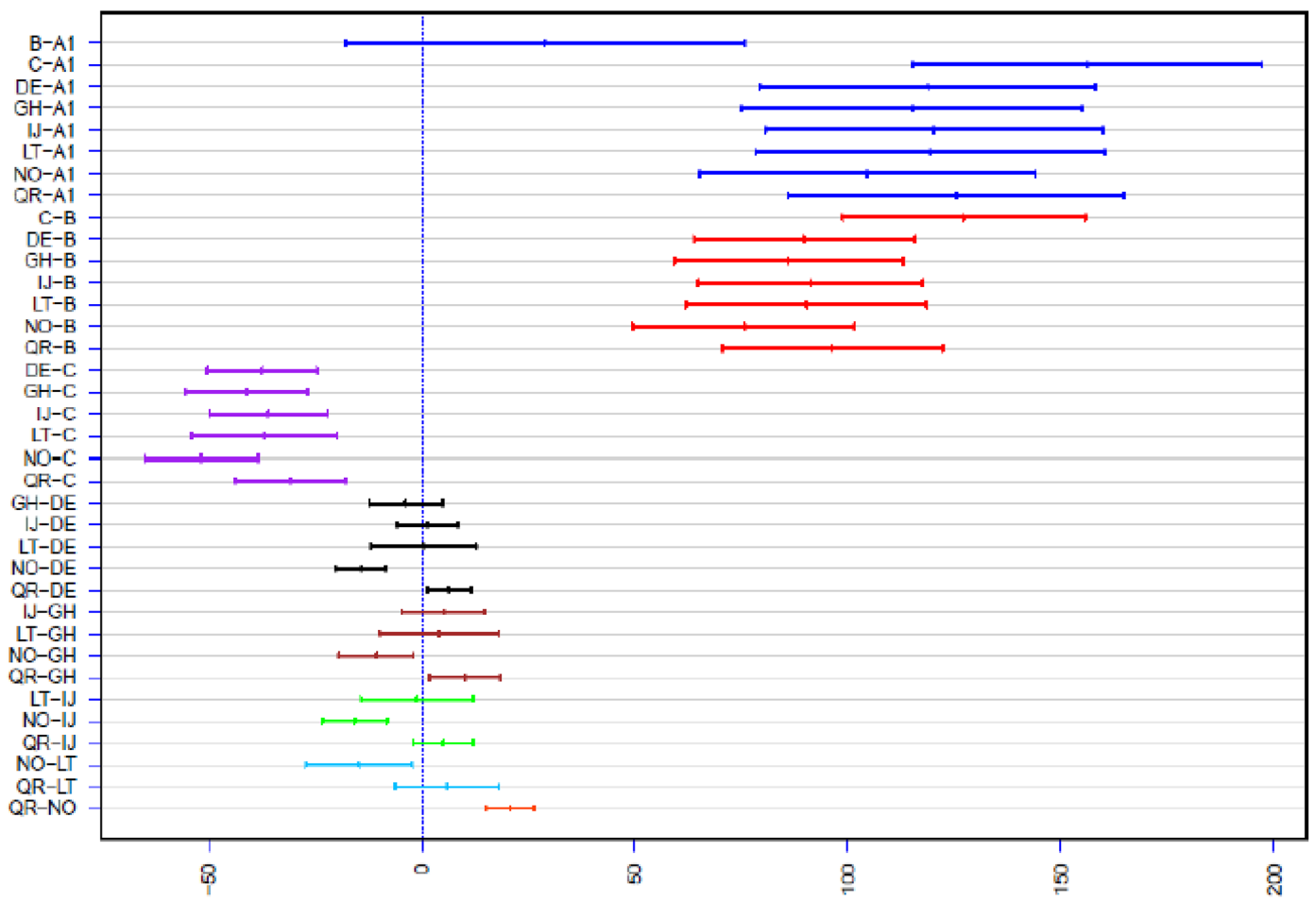

Figure 7. 95\% confidence intervals for all pair-wise divergence differences compared to the Y chromosome A1 ancestor. No significant difference exists for any bar that straddles the vertical line at zero. Bars to the left of the zero line indicate that the first group in the pair have accumulated significantly fewer mutations from the A1 ancestor than members of the second group. Bars to the right of zero indicate the members of the first group have accumulated significantly more mutations since the A1 ancestor.

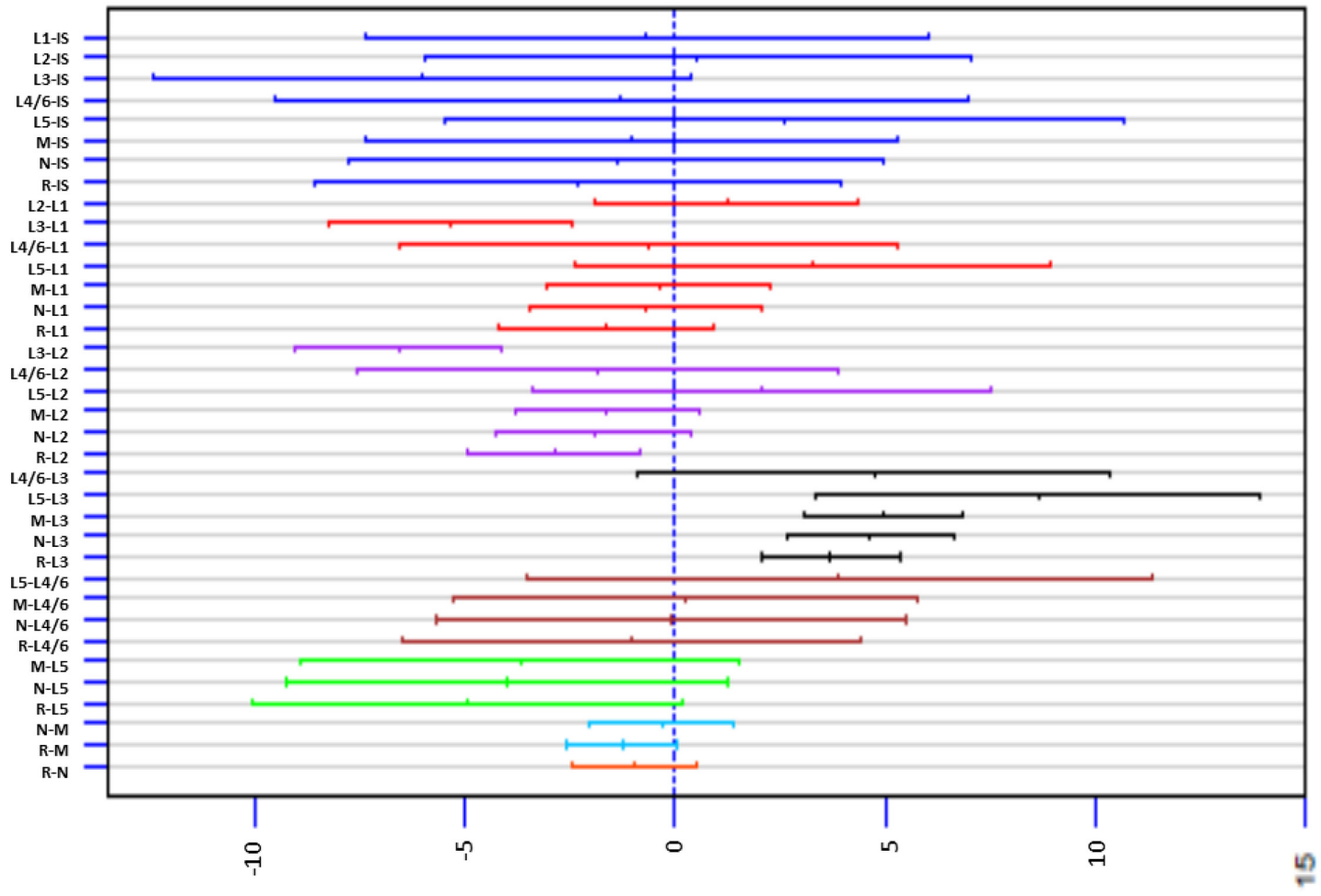

Figure 8. 95\% confidence intervals for all pair-wise divergence differences compared to the mitochondrial L0 ancestor. Similar to Fig. 7, bars to the left of the zero line indicate that the first group in the pair have accumulated significantly fewer mutations from the L0 ancestor than members of the second group. Bars to the right of zero indicate the members of the first group have accumulated significantly more mutations since the L0 ancestor. 
to be the founder of more than two major lineages, each with a uniquely definable set of mutations. The phylogenetic tree of Hallast et al. (2015) included a 3-way polytomy between the members of $\mathrm{Y}$ chromosome macrohaplogroup $\mathrm{K}$ (which includes our groups $\mathrm{L} / \mathrm{T}, \mathrm{O} / \mathrm{N}$, and $\mathrm{Q} / \mathrm{R}$ ), with three main branches, each leading to two of these haplogroups. We identified a SNV that separates $\mathrm{L} / \mathrm{T}$ from N/O/Q/R: rs2033003/M526, where an $\mathrm{A} \rightarrow \mathrm{C}$ mutation led to the latter branches. This was confirmed by Chiaroni et al. (2009), who had earlier identified this as a branch point between these haplogroups, and recently by Poznik et al. (2016). However, even though this resolved into a dichotomy between $\mathrm{L} / \mathrm{T}$ and $\mathrm{N} / \mathrm{O} / \mathrm{Q} / \mathrm{R}$, the ancestor of $\mathrm{L} / \mathrm{T}$ is but one mutation away from the ancestor of the others. This was not evident in the earlier SNV data and was only revealed with full-chromosome sequencing. The summary tree given by Poznik et al. (2016) is helpful, but they report only the most basal mutations for each branch, and so it is not always apparent how close the ancestors of these groups are.

Y chromosome haplogroups $\mathrm{G}$ and $\mathrm{H}$ are also separated by a single mutation. Poznik et al. (2013) resolved a complex 3-way polytomy previously found here. They claimed that a single substitution at rs73614810/M578, a C $\rightarrow$ T transition, separates haplogroup G from haplogroup H/I/J/K. Even more recently, Poznik et al. (2016) split the phylogeny here with variant M201 leading to $G$ and variant

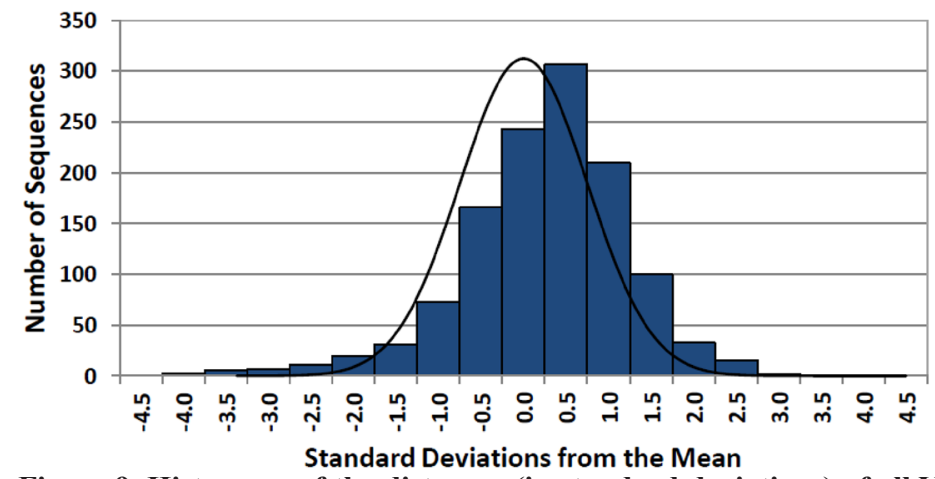

Figure 9. Histogram of the distances (in standard deviations) of all $Y$ chromosome sequences to their respective founder. The data are fitted with a normal distribution centered on 0 with a standard deviation of 1.5.

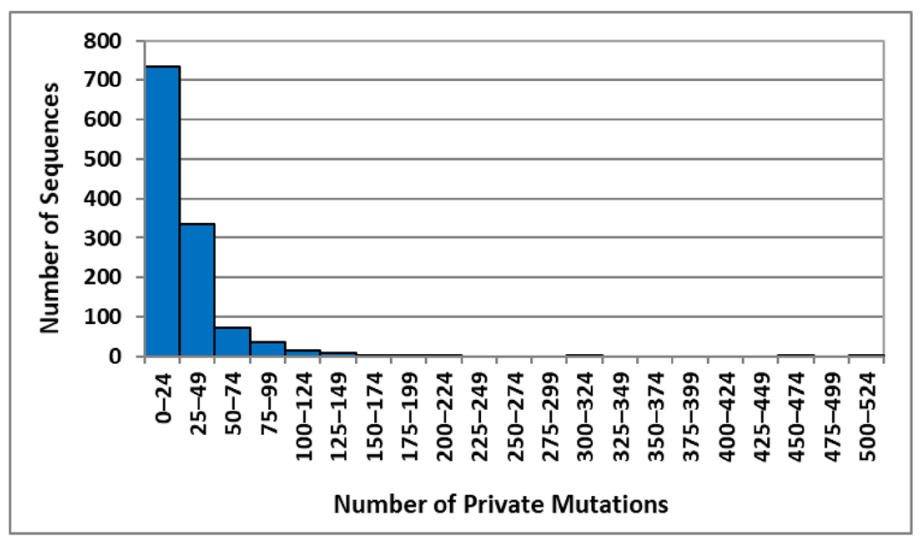

Figure 11. Histogram of all "private" $Y$ chromosome mutations. The more closely-related the individuals are in the sample, the fewer private mutations will be discovered in the data.
M578 leading to $\mathrm{H} / \mathrm{I} / \mathrm{J} / \mathrm{K}$. We also confirmed this.

The Y sequence data does not cover the entire chromosome. It is obvious that additional mutations are waiting to be observed in the not-yet-sequenced sections, and so further separation among the haplotypes might be made in the future. Likewise, we have not considering indels, inversions, duplications, etc. Including these additional features might allow for more refined clade separation. However, additional data are not expected to change the basic patterns we are seeing. It is abundantly clear that major haplogroup ancestors are closely related, as the biblical model would predict.

The mitochondrial data displays much more polytomy. This is not evident in the phylogenetic tree (Fig. 2) until one realizes the most closely-spaces branches are closer than one mutation length. In other words, they actually have zero differences. This means that multiple female lines branched off nearly instantaneously from one or a few founding females.

Unlike the situation with the Y chromosome, the mitochondrial data are complete. There are no hidden variants among the people sampled. Adding more sequences might reveal previously unknown branches in the family tree, as occurred recently with the Y chromosome (Mendez et al. 2013), but hundreds of thousands of mitochondrial sequences have been analyzed to date and so this is

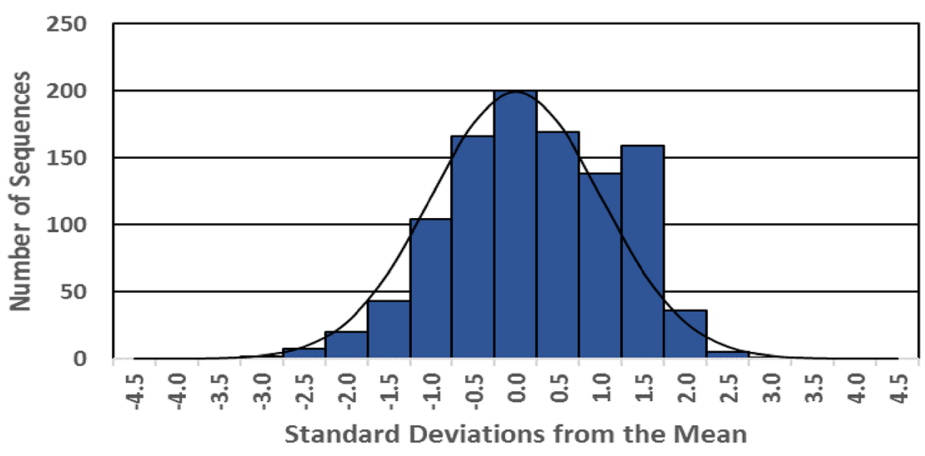

Figure 10. Histogram of the distances (in standard deviations) of all mitochondrial chromosome sequences to their respective founder.

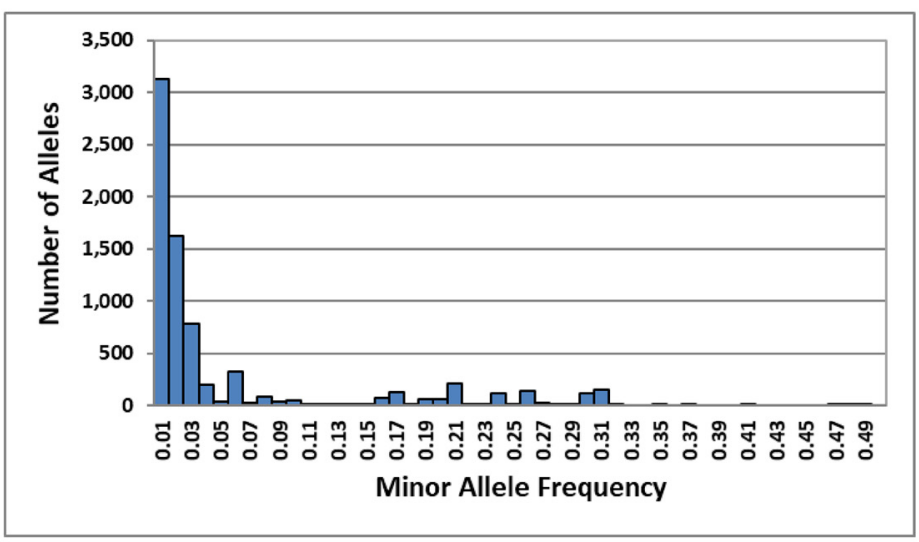

Figure 12. Y chromosome minor allele frequency histogram. 
becoming more and more unlikely.

\section{Possible causes for variable mutation accumulation rates:}

The patterns we are seeing in human lineages display some strikingly similarities with the patterns seen in the famous LTEE bacterial experiment (Tenaillon et al. 2016). A single strain/culture of $E$. coli was separated into 12 isolated lineages and their genetic divergence over time was directly observed and documented. After 50,000 generations, two clones from each of the 12 lineages were sequenced. Six of the 12 lineages picked up an average of just 43.1 mutations over the 50,000 bacterial generations. But the other six lineages experienced hypermutation, ranging from 1100 to 2500 accumulated mutations. In exactly the same amount of time, the hypermutating strains accumulated two orders of magnitude more mutations than normal.

There were two distinct patterns of mutation evident in the hypermutating strains (Table 3 ). Four of the cultures picked up a huge number of transitions of all four types, ranging from 115 to 1070 total mutations. Another two cultures picked up a huge number of $\mathrm{A} \rightarrow \mathrm{C}$ transversions (and obviously the reverse compliment $\mathrm{T} \rightarrow \mathrm{G})$. The hypermutation in the famous citrate-digesting strain (Blount et al. 2008; Barrick and Lenski 2009) has been traced to a defective MutS gene. We suspect the there were similar mutations affecting DNA repair in the other hypermutating strains. characteristic mutation accumulation patterns. We do not yet know if such patterns will be evident in the human genomic data. Preliminary analyses revealed interesting differences among the very rare African lineages, compared to all the other lineages, but this difference was not found to be statistically significant. This is an area for future research.

Gene conversion (Trombetta and Cruciani 2017) is also a possible explanation for variable mutation rates. Rozen et al. (2003) estimated that an average of 600 nucleotides per newborn male have undergone gene conversion between the two arms of the $\mathrm{Y}$ chromosome, and Trombetta et al. (2014) concluded that gene conversion between similar portions of the $\mathrm{X}$ and $\mathrm{Y}$ chromosomes is frequent. Not only does the conversion rate vary by sex and age (Halldorsson et al. 2016), but it may depend on overall heterozygosity, if it is correlated to DNA excision and repair pathways during chromosomal recombination events (Duret and Galtier 2009). The African populations are much more heterozygous than non-Africans. For example, on chr22 the 504 individuals from the four African populations were heterozygous at $3.78 \%(+/-0.20 \% \mathrm{SD})$ of all variable alleles. The 502 individuals from the five European populations were heterozygous at only $2.91 \%(+/-0.15 \% \mathrm{SD})$ of all variable sites. However, to date it is unknown if conversion is truly associated with heterozygosity in humans.

Table 3. Mutation accumulation patterns in the twelve cultures of the Long Term Evolution Experiment (LTEE). The mutation count incudes indels, inversions, mobile element insertions and losses, SNVs, etc. Hypermutating strains are shaded. Dark = the four transition-accumulating strains, including the "citrate-digesting" clone Ara-3. Light $=$ two clones that have accumulated a considerable number of $\mathrm{A} \rightarrow \mathrm{C}$, and its reverse compliment $\mathrm{T} \rightarrow \mathrm{G}$, transversions.

\begin{tabular}{|c|c|c|c|c|c|c|c|c|c|c|c|c|c|c|}
\hline \multirow{2}{*}{$\begin{array}{c}\text { Mutator } \\
\text { Status }\end{array}$} & \multirow{2}{*}{ Clone } & \multirow{2}{*}{$\begin{array}{c}\text { Mutation } \\
\text { Count }\end{array}$} & \multicolumn{4}{|c|}{ Transitions } & \multicolumn{8}{|c|}{ Transversions } \\
\hline & & & $\mathrm{AG}$ & GA & CT & $\mathrm{TC}$ & $\mathrm{AC}$ & AT & $\mathrm{CA}$ & $\mathrm{CG}$ & $\mathrm{GC}$ & GT & TA & TG \\
\hline \multirow{10}{*}{ 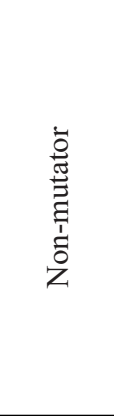 } & Ara $+2 a$ & 70 & 7 & 4 & 4 & 4 & 6 & & 5 & & 1 & 4 & & 3 \\
\hline & $\mathrm{Ara}+2 \mathrm{~b}$ & 70 & 7 & 5 & 4 & 4 & 6 & & 5 & & 1 & 4 & & 3 \\
\hline & Ara $+4 a$ & 69 & 5 & 7 & 8 & 4 & 6 & 1 & 1 & & & 2 & 4 & 3 \\
\hline & Ara $+4 b$ & 69 & 5 & 7 & 8 & 4 & 6 & 1 & 1 & & & 2 & 4 & 3 \\
\hline & Ara $+5 a$ & 79 & 10 & 4 & 12 & 2 & 3 & 1 & 3 & & & 6 & 2 & 2 \\
\hline & $\mathrm{Ara}+5 \mathrm{~b}$ & 81 & 10 & 4 & 11 & 2 & 4 & 1 & 2 & & & 5 & 2 & 3 \\
\hline & Ara-5a & 89 & 8 & 4 & 9 & 2 & 9 & 2 & 3 & & & 9 & 2 & 3 \\
\hline & Ara-5b & 94 & 9 & 3 & 10 & 3 & 9 & 2 & 2 & & & 9 & 2 & 4 \\
\hline & Ara-6a & 93 & 8 & 3 & 6 & 4 & 4 & 2 & 5 & & & 3 & 3 & 2 \\
\hline & Ara-6b & 77 & 8 & 3 & 8 & 1 & 5 & 2 & 4 & & 1 & 4 & 1 & 2 \\
\hline \multirow{12}{*}{ 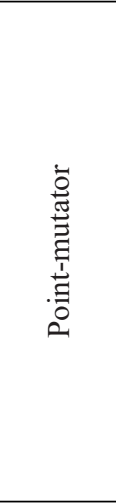 } & Ara+6a & 2595 & 10 & 5 & 6 & 3 & 1239 & 1 & 3 & 1 & & 6 & & 1311 \\
\hline & Ara $+6 b$ & 2335 & 8 & 3 & 5 & 1 & 1124 & 1 & 15 & & & 23 & 1 & 1136 \\
\hline & Ara-1a & 1112 & 7 & 2 & 6 & 4 & 511 & 2 & 17 & & & 24 & 2 & 506 \\
\hline & Ara-1b & 1135 & 8 & 2 & 7 & 4 & 521 & 2 & 16 & & & 25 & 2 & 516 \\
\hline & Ara $+3 a$ & 154 & 26 & 27 & 30 & 25 & & & 2 & 1 & & & 2 & \\
\hline & Ara $+3 b$ & 156 & 30 & 23 & 28 & 31 & & & 3 & 1 & & 1 & 2 & \\
\hline & Ara-2a & 1056 & 220 & 203 & 180 & 225 & 4 & 4 & 3 & 1 & 1 & 1 & 6 & 4 \\
\hline & Ara-2b & 1117 & 210 & 232 & 213 & 255 & 4 & 6 & 3 & 3 & 2 & & 3 & 1 \\
\hline & Ara-3a & 795 & 162 & 129 & 191 & 118 & 7 & 3 & 7 & 2 & 4 & 6 & 4 & 3 \\
\hline & Ara-3b & 822 & 183 & 135 & 177 & 116 & 7 & 3 & 11 & 4 & 4 & 11 & 5 & 2 \\
\hline & Ara-4a & 1343 & 245 & 290 & 234 & 265 & 3 & 4 & 4 & 8 & 6 & 4 & 3 & 1 \\
\hline & Ara-4b & 1362 & 248 & 290 & 238 & 262 & 6 & 4 & 4 & 8 & 6 & 4 & 4 & 2 \\
\hline \multirow{2}{*}{$\begin{array}{c}\text { IS } \\
\text { mutator }\end{array}$} & Ara $+1 a$ & 125 & 11 & 4 & 4 & 7 & 3 & & 4 & & 1 & 5 & 2 & 8 \\
\hline & Ara $+1 b$ & 128 & 11 & 5 & 3 & 5 & 3 & & 4 & & 1 & 4 & 2 & 9 \\
\hline
\end{tabular}


chromosomal recombination (Lesecque et al. 2013). Africans also have higher rates of recombination than non-Africans (Hinch et al. 2011). If gene conversion is correlated to recombination rates, this might explain some of the differences among populations we are seeing in our data.

10. Estimating the age of the primary root sequences for chrY and chrM: Given that we can reconstruct the primary root sequences for both chrY and chrM, we can very roughly estimate the age of those ancestral sequences. To do this, however, one must assume some sort of a molecular clock. Given that our data clearly shows significant differences in the rate of mutation accumulation among the different lineages, these age estimates require a large margin of error.

What is the chrY mutation rate? Using detailed genealogical knowledge, Helgason et al. (2014) reported a rate of $8.71 \times 10^{-10}$ per site, per person, per year for the Y chromosomes of a selection of Icelandic males. We must point out that modern Icelandic males are hardly an acceptable analogue for all males throughout all history. Skov et al. (2017) translated that into a rate of $3.14 \times 10^{-8}$ per site, per person, per generation (using a backcalculated generation time of 36 years) for the X-degenerate portions of the $\mathrm{Y}$ chromosome. They reported a higher rate for the heterochromatic areas and a lower rate for the ampliconic areas, but most of the 1000 Genomes data is in the X-degenerate areas so we can ignore the other sections. For comparison, Xue et al. (2009) reported a similar rate of $3.0 \times 10^{-8}$ per site, per person, per generation, but they also noted that their rate depends upon an assumed generation time and an assumption about the time to the human/chimpanzee split.

The 1000 Genomes Y chromosome data spanned 26,111,460 nucleotides on the $\mathrm{Y}$ chromosome. If we exclude any gaps greater than 2,000 nucleotides, total coverage is reduced to $10,406,614$

Table 4. Age estimates for the major Y chromosome haplogroup founders. The minimum number of generations and years are based on the work of Jeanson and Carter (2017). The maximum number of generations and years are based on Xue et al. (2009). The last row shows the average age across all haplotype ancestors. Clearly, one cannot simply apply a linear rate estimate to the distance data, especially since the different branches have had demonstrably different rates of mutation accumulation.

\begin{tabular}{|c|c|c|c|c|}
\hline Haplogroup & Min Gens & Min Years & Max Gens & Max Years \\
\hline A1 & 209 & 6,282 & 3,560 & 106,800 \\
\hline B & 137 & 4,112 & 2,330 & 69,900 \\
\hline C & 118 & 3,526 & 1,998 & 59,950 \\
\hline D/E & 111 & 3,331 & 1,887 & 56,625 \\
\hline G & 77 & 2,313 & 1,311 & 39,326 \\
\hline H & 79 & 2,377 & 1,347 & 40,406 \\
\hline I/J & 79 & 2,372 & 1,344 & 40,332 \\
\hline L/T & 76 & 2,280 & 1,292 & 38,757 \\
\hline N/O & 73 & 2,183 & 1,237 & 37,105 \\
\hline Q/R & 77 & 2,319 & 1,314 & 39,428 \\
\hline Average & 104 & 3,110 & 1,810 & 52,900 \\
\hline & & & & \\
\hline
\end{tabular}

nucleotides. Since most gaps are very large, increasing the cutoff to $>=10,000$ nucleotide gaps has little appreciable effect on total coverage (the total span increases by only $1.5 \%$ ).

The age estimates for each chrY haplogroup ancestor, using two vastly different rate estimates, are shown in Table 4. Clearly, it is not possible to simply scale the data linearly. Our discovery that mutation rates are not constant among the haplogroups solves this dilemma and allows us to explore alternatives without being held back by evolutionary molecular clock assumption.

What is the chrM mutation rate? Quoted mitochondrial mutation rates can be quite variable and depend on method (phylogeny vs. genealogy), area sampled (e.g., hypervariable region vs. total chromosome) and type of mutations studied (synonymous vs. nonsynonymous, coding vs. non-coding, etc.). The lack of a standard measure is a well-known problem in molecular clock estimates in mitochondrial studies (Loogväli et al. 2009). Yet, we are not concerned with the absolute rate so much as an approximation. If widely-discordant evolutionary rates match a general biblical timeframe, there is little need to attempt to determine the exact rate. Plus, once we discovered that mutation rates can vary significantly from one group to another, we realized that a single rate that can be applied to all of humanity across our entire history should not exist. For example, Soares et al. (2009) attempted to divide the mitochondrial genome into eight fractions and calculate an overall expected mutation rate. Their figure of $1.7 \times 10^{-8}$ mutations per site, per year (or one mutation every 3,624 years) was ridiculously low and is entirely influenced by the assumed human-chimp split time of 6.5 MA. We reject all phylogenetic mutation rates as unrealistic. On the other end of the spectrum, Madrigal et al. (2012) used a genealogical method to measure a rate of $1.24 \times 10^{-6}$ per site, per year in the second hypervariable section (HVSII). This amounts to approximately 1 mutation every-other generation, after accounting

Table 5. Age estimates for the major mitochondrial chromosome haplogroup founders. The minimum number of generations and years are based on Madrigal et al. (2012). The maximum number of generations and years are based on Sigurðardóttir et al. (2000). As with the Y chromosome data, these estimates cover a huge range, reflecting a large margin of error. The last row averages across all haplogroup founders. Since mutation rates are not consistent, it is not actually appropriate to apply a linear rate estimate, but we do so here to illustrate the difficulties anyone has in assigning dates to these historical events.

\begin{tabular}{|c|c|c|c|c|}
\hline Haplogroup & Min Gens & Min Years & Max Gens & Max Years \\
\hline L1 & 153 & 4,603 & 1,245 & 37,336 \\
\hline L5 & 152 & 4,565 & 1,234 & 37,027 \\
\hline L2 & 126 & 3,781 & 1,022 & 30,669 \\
\hline L4/6 & 91 & 2,745 & 742 & 22,264 \\
\hline L3 & 72 & 2,161 & 584 & 17,526 \\
\hline M & 87 & 2,612 & 706 & 21,183 \\
\hline I/S & 81 & 2,429 & 657 & 19,699 \\
\hline N & 75 & 2,263 & 612 & 18,359 \\
\hline R & 67 & 2,013 & 544 & 16,324 \\
\hline Average & 100 & 3,020 & 816 & 24,500 \\
\hline
\end{tabular}


for the size differences and mutation rate differences of HVSII compared to the rest of the mitochondrial genome. Sigurðaróttir et al. (2000) calculated a rate of 0.0043 per generation for the entire mitochondrial control region. We can extrapolate the total number of mutations expected across the entire molecule without having to partition the data like Soares et al. (2009) did. We found 25.3 times more mutations genome-wide than in HVSII and 8.5 times more mutations genome-wide than in the control region. Taking this and applying it to the rate estimates in these two studies allowed us to estimate when each of the major haplogroup ancestors lived, with a purposefully large degree of uncertainty. As in the Y chromosome estimates above, the mitochondrial estimates varied widely among haplogroups and among the two rate estimates (Table 5).

\section{Discussion}

For years geneticists have known that there is a single paternal ancestor and a single maternal ancestor for all of humanity (Cann et al. 1987; Karafet et al. 2008). This is a direct prediction of the biblical model. It can also be explained in the evolutionary model, but only by assuming random mating on a global scale, and by invoking a bottleneck that would in any other species almost certainly cause extinction. The evolutionary model did not anticipate this discovery. Instead, the evolutionary model had to be radically modified to accommodate this remarkable development while invoking various ad hoc rescue mechanisms, specifically a long-term bottleneck among the African population. It is widely known that the inbreeding effects of any serious population bottleneck are deleterious, and having an effective population size of just a few thousand individuals for many thousands of years would cause population degeneration and population collapse, not radical evolutionary advance and explosive growth into all corners of the world.

\section{We now know the sequence of each founding ancestor of} each major lineage in both the chrY and chrM family trees.

It is possible to reconstruct the actual ancestral sequence at any node in either the human chrY or chrM ancestry tree. This is not a theoretical construction, but a valid recreation of the original chromosome of historical individuals. The biblical patriarchs are in those trees, but they may or may not sit at one of these nodes. We cannot assume there should be 16 branches for the chrY tree, for example, just because Noah had 16 grandsons (actually, many of the names in the Table of Nations in Genesis 10 are plural, indicting people groups rather than named individuals). Population dynamics are complex. Some lineages have doubtlessly thrived and multiplied, while some lineages have doubtlessly diminished and been lost.

Not only do we actually know the sequences of these haplotype "patriarchs" and "matriarchs", we can roughly approximate the time when they lived. In the same way, we can roughly approximate the time when the singular paternal patriarch (Adam/Noah) and the singular maternal patriarch (Eve) lived. Given a few reasonable assumptions, we obtain similar ages for both the primary male ancestor, and primary female ancestor. These dates have a wide margin of error, but on the low end they are very much closer to the expected biblical age than the expected evolutionary age (see tables 3 and 4 under results).

\section{Approximation of the primary root sequences for chrY and chrM}

Even though the phylogenetic trees displayed a bold, star-like pattern, with the majority of lineages radiating from a central area, it is still difficult to pin down the exact location of the ancestral sequence in either tree. The evolutionists have an advantage here in that they use chimpanzee as a rooting lineage and assume that the ancestor is the most chimp-like of the sequences because any location with a chimpanzee reading is assumed to carry the ancestral allele. This does not mean that they believe some people are more closely-related to chimps than others but that some lineages branched off earlier.

In the biblical model, however, we do not know if we are starting with a single chrY lineage or if the sons of Noah were quite dissimilar. The same is true of chrM. The Bible says nothing about how closely related the three daughters-in-law were to one another, so we do not know what to expect at the root of the tree. For these reasons, it is not possible to precisely identify the primary root sequences for either chrY or chrM, but we can still make good approximations, within a reasonable margin of error.

From first principles, we would place Y Chromosome Adam/Noah somewhere along the branch between junction of haplogroups A through $\mathrm{E}$ and the root of macrohaplogroup F. Comparing the distribution of haplogroups A through $\mathrm{E}$ makes us conclude that the major ancestral node of these groups could quite possibly represent the Y chromosome of Ham. Since many Jewish men carry chrY haplogroup J (the naming of these groups had nothing to do with religious identity; "J" for Jewish is a fluke), and since they descend from Shem (but see Carter 2017), the root of haplogroups G, H, I, and $\mathrm{J}$ is a good candidate for the $\mathrm{Y}$ chromosome of Shem. Japheth would, then, be located at the root of the remaining haplogroups. But, several other possibilities exist. Even so, the number of mutations separating the major haplogroup ancestors is still not necessarily equal to the time that separates them.

The placement of Mitochondrial Eve also has a large margin of error. However, a good candidate location would be at the junction of haplogroups $\mathrm{L}$ and $\mathrm{M}$. Whether or not the three daughters-in-law shared the same mtDNA or different is a matter of speculation. In our earlier work (Carter et al. 2008) we identified the ancestor of macrohaplogroup $\mathrm{R}$ as the "Eve" sequence. This was a tentative conclusion and we directly stated that further modifications of that placement were possible, with the most likely scenario involving moving Eve to macrohaplogroup $\mathrm{N}$ or even beyond it toward haplogroup L3. Here we would like to suggest that Eve is most likely to be located along the long branch that connects macrohaplogroup L/M to macrohaplogroup $\mathrm{N}$. The daughters-inlaw, therefore, would be arrayed around her, leading to the major starburst pattern seen in the data. Alternatively, she could represent the L/M ancestor. Remember, if any of the daughter-in-law were sisters, we could be starting off with fewer than three major lineages.

The evolutionary model is not nearly as clear-cut as many believe. For example, in 2013 a major new branch was added to the Y chromosome tree (Mendez et al. 2013). This new line was discovered by a genealogy testing company and resulted in a 
great expansion of the date for (the evolutionary) Y Chromosome Adam. In fact, it brought it to within a similar date range for (the evolutionary) Mitochondrial Eve. While some have argued that the exact date was a bit off (Elhaic et al. 2014), the point is that a single new discovery caused a radical redating of an established evolutionary ancestor. We need to point out that this new haplogroup (A00) is extremely rare. Additional men living in Cameroon have been found that belong to this group, but the fact is that rare lineages tend to not persist over deep time. The more rare a variant is, the more likely it is to be lost to drift. Do these men really represent an extremely old branch that managed to persist in an out-of-the-way corner of Africa, or are they from a much newer branch that more recently experienced an elevated mutation rate? The fact that they carry the "ancestral" allele at multiple positions is taken as proof that they are from an older branch. But, since one out of three SNVs at places where humans and chimpanzees differ will result in the assumed "ancestral" allele, any highly mutated branch will naturally fall into that pattern, even if they share no common ancestry.

\section{Patriarchal drive?}

For both chrY and chrM, certain lineages have picked up more mutations compared to others in the same amount of time (Moorjani et al. 2016). The reason for this is unknown. Population size (Krašovec et al. 2017), overall heterozygosity (Yang et al. 2015), and the presence of known mutagens in the environment, such as surface rocks containing high amounts of thorium (Forster et al. 2007), can affect mutation accumulation. But genetic factors such as the frequency of recombination (Hinch et al. 2011) and the presence of defective or directional repair enzyme systems (Pinto et al. 2016) can also play a role. It is known that mutation rates vary from one family to another (Conrad et al. 2011; Rahbari et al. 2015), due to genetic factors.

We know that mutations accumulate like clockwork in some genetic systems, even given strong natural selection (Carter and Sanford 2012), and the types of mutations can be predictable (Carter 2014). The reason for this is that certain chemical reactions are more likely than others. Thus, the spontaneous deamination of methylated $\mathrm{C}$ in $\mathrm{CpG}$ nucleotide pairs leads to recurrent and frequent $\mathrm{C} \rightarrow \mathrm{T}$ mutations in eukaryotes.

We also know there is an age effect. It is known that as people grow older, their reproductive cells accumulate more mutations. This is especially true for males (Crow 1997; Kong et al. 2012; Francioli et al. 2015). In the biblical model, the patriarchs grew to be exceedingly old. The very old patriarchs would have contributed a huge number of new mutations to their children, assuming similar rates of cell division and polymerase-induced mutation in the gonads as seen today. We note that Noah is the oldest father recorded in the Scriptures. Because of this, Shem, Ham, and Japheth could have received a huge number of new mutations, and so it is possible that each son could have established, in a single generation, a substantial new branch on the phylogenetic tree.

Also, the paternal age-effect appears to be non-linear (Crow 1997), perhaps even exponential, meaning that very old men having children late in life could have instantaneously created brand new lines on the family tree. These lines are assumed, by most, to be the result of slow mutation accumulation over time, but biblically we have reasons to reject this uniformitarian assumption. Instead, we would like to introduce the term "patriarchal drive" to indicate the genetic, demographic, and mutational effects inherent in a biblical model with centuries-old people as both founders and long-term residents within that population, who continue to have children until late in life. For most of human history, normal population genetics and demographics would apply. But this is not true in the early years of biblical history.

\section{Out of Babel, not Out of Africa}

The general pattern of what we see in both Y chromosomes and mitochondrial DNA supports a single primary dispersion of humanity in the recent past. Some call this dispersion "Out of Africa". We call it "Out of Babel".

The data reveals an interesting pattern, in that multiple major branches have arisen from surprisingly closely related individuals, in very short windows of time. In both trees, multiple major branches can be traced to identical ancestral individuals. These could be brothers/sisters or cousins. If the mutation rate for chrY and chrM is less than one per generation, it might not be possible to capture all lineage-forming events, but the fact remains that the individuals who gave rise to the major clades were not far apart in time. This is not at all feasible in the evolutionary model. The chances are vanishingly small that in a large population any two closely-related people would go on to have millions of ancestors. Yet this is exactly what we see happening, many times, in both of the chrY and chrM trees. The credible way to explain this it that an explosive population expansion happened early in human history, starting with just a few families or small tribes. This does not fit well with the evolutionary model, and evolutionary ideas of human demography, but it naturally falls out of the biblical model with rapid growth from a single small population (Carter and Hardy 2015; Carter and Powell 2016).

In order for history to capture multiple major branches that trace back to very closely related individuals, humanity must have gone through an extreme population bottleneck followed by explosive population growth, as concluded by other studies (Keenan and Clark 2012). This is the exact scenario one would predict in a Flood/Babel model.

Nearly all major group ancestors seem to trace back to the Middle East. The most common Y chromosome haplotype in Africa today (E) apparently arose outside of Africa (Karmin et al. 2015; Poznik et al. 2016). This makes the Out-of-Africa theory even more problematic.

\section{Polytomy reveals much about human history}

Another indication of the rapid formation of both the chrY and chrM major haplogroups is the presence of multiple polytomies. As data density has increased from SNV data to fully sequenced chromosomes, most polytomies have been resolved. However, the remaining ones are extremely problematic for evolutionary theory, and "near-polytomies" (arising almost simultaneously) are more common and are almost as problematic.

\section{Violations of the molecular clock hypothesis}

The molecular clock might be applicable in certain situations where the reproducing entity is simple and only a few individuals 
manage to propagate their genes from one year to the next (e.g., the human H1N1 influenza virus, see Carter and Sanford [2012] and Carter [2014]). Alternatively, molecular clocks might work in a broad sense when one is able to average accumulation rates among diverse lineages (e.g., Jeanson 2015a). But when this is done, the rates generally line up with the biblical timeframe and defy evolutionary long ages (Jeanson 2013, 2015b; Tomkins 2015). Also, several authors have called for rate variation during human history, specifically a higher recombination rate within Africans (e.g., Jeanson 2016; see also Hinch et al. 2011) or a higher rate associated with the Flood or early post-Flood period (e.g., Wood 2012, 2013).

We have demonstrated that the mutation rates along various branches of the chrY and chM trees are clearly variable, manifesting statistically significant differences among multiple group-pairs. This is a direct challenge to the molecular clock hypothesis, and thus the Out of Africa theory. We conclude that either: 1) Ancestor A1 (chrY) and L0 (chrM) are not the common ancestor of these individuals; or 2) the rate of mutational divergence is not constant among haplogroups; or 3) both. Lastly, the observation that most chrY and chrM mutations are rare is excellent evidence that the human genome is young, irrespective of whether or not the clock is precise.

Lineages that have accumulated an inordinate number of mutations may have experienced innately higher mutation (similar to the mutator bacterial strains in the famous LTEE experiment), or those lineages may have had a historical episode of accelerated mutation due to environmental, epigenetic, or demographic factors. Costello et al. (2013) claimed that oxidative damage to DNA leads to artifacts in sequence data. Chen et al. (2017) concluded that the majority of $\mathrm{G} \rightarrow \mathrm{T}$ transversions in the 1000 Genomes were erroneous. Thus, there may also be artifacts in the sequencing data, perhaps even tracing back to field collection techniques.

Recently, Moorjani et al. (2016) detected violations of the molecular clock hypothesis among ten primate species. They concluded that substitution rates are higher in New World monkeys than they are in Old World monkeys and that these in turn are higher than in apes and humans. In fact, they determined that the rate was about $7 \%$ faster in gorillas and about $2 \%$ faster in chimpanzees than in humans, using nothing but evolutionary assumptions. But there is also evidence of clock violations within humans. Behar et al. (2012) found statistically significant violations of the molecular clock hypothesis for a select few mitochondrial haplogroups (M, specifically). They noted that even young haplogroups showed significant differences in terms of mutation accumulation rates. Scozzarri et al. (2014) reported a statistically significant molecular clock anomaly in terms of the mutations that led to Y chromosome haplogroup A1b. We could not verify this because none of the 1000 Genomes Y chromosomes were from that haplogroup. Mallik et al. (2016) claimed to discover an approximately 5\% increase in the rate of mutation accumulation in non-Africans over Africans, genome-wide. After factoring in the source of DNA (e.g., lymphoblastoid cell-lines, blood, and/or buccal samples) Hallast et al. (2015) concluded that subtle haplogroup-specific effects on Y-chromosome branch length do exist. Finally, Sayres et al. (2014) concluded that purifying selection (and possibly positive selection) has had a strong role in reducing Y chromosome diversity worldwide, but their study included only 16 chromosome sequences. Even though each of these studies suggested that branch lengths vary, Hallast et al. (2015) expected the effect on the time to the most recent common ancestor (TMRCA) calculation would be minor. We disagree.

We detected different amounts of mutation accumulation among the various haplogroups, which would normally be attributed to different ages of the founders. However, even haplogroups having a common ancestor (i.e., Y chromosome haplogroups N/O and $\mathrm{Q} / \mathrm{R}$ ) often had different rates of divergence (on the order of $5-10 \%)$ from that ancestor. This is a violation of the molecular clock principle. There are various explanations for this, including natural selection, but only a minority of SNVs are expected to be subject to selection (Poznik et al. 2016). Early strong population structure (in evolutionary models) could also have an influence on the shape of the modern phylogenetic tree, but how much of an effect remains an open question (Karmin et al. 2015).

Mallik et al. (2016) concluded that the mutation rate outside of Africa has been historically higher than the mutation rate within Africa, and Henn et al. (2015) claimed that mutational load increases with distance from Africa due to reduced selection. However, there are exceptions among the non-African groups as well. For example, even though N/O and $Q / R$ had an identical founder sequence, these two groups had different average ranks (in terms of divergence from their mutual ancestor). The N/O individuals consistently ranked in the first half of the distribution (less diverged) and the $\mathrm{Q} / \mathrm{R}$ individuals consistently ranking in the second half of the distribution (more diverged). Therefore, it appears that while the average rate of mutational divergence was more or less constant within haplogroups (Fig. 5), the rate of mutational divergence was variable among haplogroups (Fig. 6). This contradicts evolutionary conclusions regarding the timing of events based on the molecular clock hypothesis. Lenski's LTEE (Tenaillon et al. 2016) tells us there is a real possibility that mutator strains can emerge when sub-populations are cut off from the outside and restricted in size. We suspect the differences occurred early on in post-Flood human history and were driven by a drastically small population moving into new areas and remaining small. This may help to explain why a few scattered individuals and rare groups have highly divergent haplotypes. The same concept might explain the ancient and highly divergent Homo populations such as Neanderthals, Denisovans, $H$. erectus, H. floresiensis, and Homo naledi (we do not have DNA sequences for all of these yet, but see Wood 2012).

It should be noted that the genomes of ancient Egyptians were much more similar to those of Eurasia than sub-Saharan Africa. Substantial mixing across the Sahara has occurred, but probably not until after the rise of Islam (Schuenemann et al. 2017). In a similar way, the first people in Eurasia (apparently, Neanderthals and Denisovans) were different from those that came later. There is additional evidence that the most ancient people in southern and southeastern Africa were different from the people living there today, with the ancient genomes corresponding to the isolated and more divergent Khoi-San peoples instead of the dominant Bantus (Schlebusch et al. 2017; Skogland et al. 2017). Thus, aDNA can reveal interesting historical demographic shifts, but it is as if the 
marginal populations (e.g., Neanderthals and Denisovans, and to a much lesser extent other isolated modern populations) experienced elevated mutation rates. This is another area for further research.

Not only does evidence for historical rate variation in different lineages exist, but it is also clear that the dates given for divergence events are not independently derived. For example, the split between $\mathrm{Y}$ chromosome haplogroups $\mathrm{Q}$ and $\mathrm{M} 3$, an important event that is supposed to have occurred just prior to the peopling of the Americas, is estimated to have happened 15 KYA. Behar et al. (2012) and Poznik et al. (2016) claimed this "provides a sanity check" for clock calibrations. Clearly, they are prepared to reject measured mutation rates in favor of evolutionary assumptions if the measured rates turn out to be too high.

\section{Not all mutations are independent}

Recurrent mutations are a real concern. We detected hundreds of repetitive mutations on the mitochondrial chromosome and thousands on the $\mathrm{Y}$ chromosome. Approximately half of the existing variations on chromosome 22 are $\mathrm{C} \rightarrow \mathrm{T}$ (and its reverse compliment $\mathrm{G} \rightarrow \mathrm{A}$ ). And more than half of those occur at $\mathrm{CpG}$ sites, a classic site for epigenetic modification. It is possible that anomalous ancient DNA and the extremely divergent modern lineages represent environmental effects, that is, epigenetic modifications that accidentally get hardwired by the loss of the cytosine through deamination. It is also possible that independent lineages could pick up identical mutations over time due to environmental effects. Because so many mutations have occurred at $\mathrm{CpG}$ sites, there has been a huge change in epigenetic control over the years. This might have something to do with the loss of longevity in the early post-Flood population. This is yet another area calling for additional research.

\section{Further considerations}

There are several additional caveats that we must consider. Genetics is an imperfect science and we are delving deep into human history, sometimes with insufficient data. Because of this, we do not know the precise location of the chrY and chrM roots. Due to greater phylogeographic uncertainty near the root of the tree (Scozzari et al. 2014) we cannot precisely know just how closely related those individuals were, nor how much time separated them. Early populations are expected to experience strong drift due to small population sizes, and they are not likely to stay in one geographic location for long periods of time. Yet, it is clear from our study that many of the major haplogroup ancestors were closely related to one another. This is also obvious from the phylogenetic trees of many earlier studies, but the significance of this appears to have been missed by those authors. In this paper, we have carefully documented the many polytomies and near-polytomies in both trees, and we have demonstrated the implications of this: individual families or small tribes grew explosively, simultaneously giving rise to multiple lineages of major importance. This is reminiscent of Genesis 10 and 11, where a single family grew into many tribes, nations and languages.

We must remember that there has been much replacement of older haplotypes during human history. The men of haplogroup $\mathrm{E}$ had to migrate from Asia into Africa, quickly growing to becoming the dominant haplogroup on that continent (Poznik et al 2016).
This appears to be a greater population expansion than the more recent Bantu expansion (Campbell and Tishkoff 2010), which also carried $\mathrm{E}$ along with it. And, even though haplogroup $\mathrm{R}$ is common in Eurasia, and even though its roots appear to be in Central Asia, this group also expanded into Africa, penetrating as far south as Cameroon (Chiaroni et al. 2009). The dominant haplogroups today clearly did not expand into uninhabited territory (Slatkin and Racimo 2016).

The Table of Nations (Genesis 10) was a one-off documentation of the early post-Flood world, with about 4,0000 years of history since. Much more work needs to be done before we can claim to precisely identify any of the biblical patriarchs. In the end, the data preserved among people living today, and among our ancestors buried in graves worldwide, may not give us a perfect recreation of history. What we can see already, however, is consistent with a generally straightforward reading of Genesis.

We would like to note that the original Eve sequence (Carter 2007; Carter et al. 2008) was based on a consensus, which got us close enough at that time to draw several significant conclusions about human history. Bandelt et al. (2014) cited that earlier work, directly misconstruing our methods while taking a swipe at "creationism". They apparently did not read either of those papers. Wood (2012) was more charitable, but he seems to have missed the fact that we were not saying Eve1.0 was the historical mtDNA source. To be clear, it is not possible to construct an ancestral sequence with zero ambiguity. The methods we employ here get us just a little closer to the primary root sequence ("the Eve sequence") than where we were in those earlier papers. However, in this paper we have determined the exact sequences of all of the chrM haplogroup and megahaplogroup founders, and we have reconstructed the chrM phylogenetic tree, which reveals major molecular clock anomalies, and many polytomies and near-polytomies.

While this paper strengthens and expands upon our earlier chrM papers, it is also breaking new ground in terms of the investigating the history of chrY from a biblical perspective. Our investigations have revealed many surprising results, including major molecular clock anomalies, many polytomies and near-polytomies, and the exact chrY sequences of all the founders of all the major haplogroups and megahaplogroups.

The fact that the Y and mitochondrial chromosomes show similar patterns might indicate that we might be looking at post-Flood demographic effects and not the three sons of Noah vs. their three wives. Thus, it might not be possible to locate the Ark passengers on the phylogenetic tree. Finally, data quality is always a concern. Some of the branch tips are longer than they should be due to false positives, but this is maybe $1 \%$ of the modern data. However, this is a huge concern with ancient DNA (c.f. the revealing nature of fig. S11 in Haber et al. 2017), and so we urge people do be cautious about ancient DNA sequencing studies. There has been some contention among creationists on this topic, with the majority probably being on the more skeptical side. Thomas and Tomkins (2014) discussed the relevant problems and pitfalls of ancient DNA work, and there are many. However, with the publication of multiple ancient genomes to date (c.f. Yang and Fu 2018), including several Neanderthal individuals who were more similar 
to one another than to non-Neanderthals, as well as genome data from the enigmatic Denisovans (Reich et al. 2010), thus validating the original Neanderthal sequencing project, it appears that many of the major objections are slowly being answered.

Why do some haplogroups display less divergence than other groups, even though they arose simultaneously? Heterotachy (changes in site-specific mutation rates over time, perhaps due to functional divergence) has been implicated as an important process in protein evolution (Lopez et al. 2002). Yet, the majority of the sites in the $\mathrm{Y}$ chromosome are not in protein-coding regions, so functional divergence does not seem applicable here. Contemporary mutation rates may be higher in one group than another, which is unlikely since they often live among one another within the same populations, that is, with the same genetic and environmental backgrounds. For example, Poznik et al. (2016) noted that the similar patterns seen among $\mathrm{Y}$ chromosome haplogroups E1b, $\mathrm{R} 1 \mathrm{a}$ and R1b are probably due to shared historical demography. Alternatively, rates may have been higher in one group historically, but this is also difficult because that would mean there was a time when the members of both haplogroups lived in separate places and under different conditions.

We do not yet know why some populations appear to be accumulating mutations faster than others. Elevated mutation accumulation in man is an important concern in terms of human health and longevity, and so it will be important to study what factors may have historically affected mutation accumulation rates in different human populations. Factors that might influence the rate of mutation accumulation in a human subpopulation over time would include genetics, epigenetics, environment, culture, and demography. Hallast et al. (2015) also discussed these factors. We would add patriarchal drive as an important contributor to this list.

\section{CONCLUSION}

Strict Darwinists and theistic evolutionists both claim the biblical Adam and Eve never existed. However, after carefully considering the information provided here, their case is significantly weakened. In fact, if the Bible were not true, one would never expect such a strong concordance between biblical and phylogenetic history, as we have shown.

Using nearly-complete, whole-chromosome SNV data, we have calculated the founder sequences of the major haplogroups and megahaplogroups of both chrY and chrM. Our founder sequences are consistent with previous analyses that were based on more limited data (e.g., small numbers of SNVs or STRs), as well as more recent studies on whole-chromosome data. We have also created unrooted phylogenetic trees for both chrY and chrM. We show that most of the haplogroup founders tightly cluster within a star-like phylogeny. These trees and our additional analyses show that multiple haplogroup founders were surprisingly closely related, suggesting a small population that underwent explosive population growth, giving rise to all the human chrY and chrM haplogroups in the world. This conclusion is supported by the fact that we see several full or near polytomies in both phylogenies.

Within all the haplogroups, the pattern of divergence from the haplogroup founder reflects systematic mutation accumulation consistent with an imprecise molecular clock. There appears to be substantial variance in mutation accumulation rates between haplogroups, especially early in human history, which could lead to anomalies in molecular clock estimates.

In the end, there is no reason to reject a literal, historical Adam and Eve. The genetic data are pointing strongly in that direction. In fact, the data we see are exactly what we would expect from the biblical account of human origins.

\section{ACKNOWLEDGEMENTS}

This work was supported in part by FMS Foundation. Thanks for the helpful suggestions of several anonymous reviewers.

\section{REFERENCES}

1000 Genomes Project Consortium. 2010. A map of human genome variation from population-scale sequencing. Nature 467:1061-1073.

Bandelt, H.J., A. Kloss-Brandstätter, M.E. Richards, Y.G. Yao, and I. Logan. 2014. The case for the continuing use of the revised Cambridge Reference Sequence (rCRS) and the standardization of notation in human mitochondrial DNA studies. Journal of Human Genetics 59:6677.

Behar, D.M., M. van Oven, S. Rosset, M. Metspalu, E.V. Loogväli, N.M. Silva, T. Kivisild, A. Torroni, and R. Villems. 2012. A Copernican reassessment of the human mitochondrial DNA tree from its root. American Journal of Human Genetics 90:675-684.

Barrick, J.E., and R.E. Lenski. 2009. Genome-wide mutational diversity in an evolving population of Escherichia coli. Cold Spring Harbor Symposium on Quantitative Biology 74:119-129.

Blein, S., C. Bardel, V. Danjean, L. McGuffog, S. Healey, and D. Barrowdale. 2015. An original phylogenetic approach identified mitochondrial haplogroup T1al as inversely associated with breast cancer risk in BRCA2 mutation carriers. Breast Cancer Research 17:61.

Blount, Z.D., C.Z. Borland, and R.E. Lenski. 2008. Historical contingency and the evolution of a key innovation in an experimental population of Escherichia coli. Proceedings of the National Academy of Science 105, no. 23:7899-7906.

Campbell, M.C., and S. A. Tishkoff. 2010. The evolution of human genetic and phenotypic variation in Africa. Current Biology 20, R166-R173.

Cann, R.L., M. Stoneking, and A.C. Wilson. 1987. Mitochondrial DNA and human evolution. Nature 325:31-36,

Carter, R.W. 2007. Mitochondrial diversity within modern human populations. Nucleic Acids Research 35, no. 9:3039-3045.

Carter, R.W. 2014. More evidence for the reality of genetic entropy. Journal of Creation 28, no. 1:16-17.

Carter, R.W., 2017. Extensive mixing among Israelites and non-Israelites in biblical history. Journal of Creation 31, no. 3:75-81.

Carter, R.W., D. Criswell, and J. Sanford. 2008. The "Eve" mitochondrial consensus sequence. In Proceedings of the Sixth International Conference on Creationism, ed. A.A. Snelling, pp. 111-116. Pittsburgh, Pennsylvania: Creation Science Fellowship.

Carter, R., and C. Hardy. 2015. Modelling biblical human population growth. Journal of Creation 29, no. 1:72-79.

Carter, R.W. and J.C. Sanford. 2012. A new look at an old virus: Mutation accumulation in the human H1N1 influenza virus since 1918. Theoretical Biology and Medical Modelling 9:42.

Carter, R.W., and M. Powell. 2016. The genetic effects of the population bottleneck associated with the Genesis Flood. Journal of Creation 30, no. 2:102-111. 
Chen, L., P. Liu, T.C. Evans Jr., and L.M. Ettwiller. 2017. DNA damage is a pervasive cause of sequencing errors, directly confounding variant identification. Science 355:752-756.

Chiaroni, J., P.A. Underhill, and L.L. Cavalli-Sforza. 2009. Y chromosome diversity, human expansion, drift, and cultural evolution. Proceedings of the National Academy of Science 106, no. 48:20174-20179.

Conrad, D.F., J.E.M. Keebler, M.A. DePristo, S.J. Lindsay, Y. Zhang, F. Cassals, Y. Idaghdour, C.L. Hartl, C. Torroja, K.V. Garimella, M. Zilversmit, R. Cartwright, G. Rouleau, M. Daly, E.A. Stone, M.E. Hurles, P. Awadalla, and 1000 Genomes Project. 2011. Variation in genome-wide mutation rates within and between human families Nature Genetics 43, no. 7:712-714.

Costello, M., T.J. Pugh, T.J. Fennell, C. Stewart, L. Lichtenstein, J.C. Meldrim, J.L. Fostel, D.C. Friedrich, D. Perrin, D. Dionne, S. Kim, S.B. Gabriel, E.S. Lander, S. Fisher, and G. Getz. 2013. Discovery and characterization of artifactual mutations in deep coverage targeted capture sequencing data due to oxidative DNA damage during sample preparation. Nucleic Acids Research 41, no. 6:67.

Crow, J.F. 1997. The high spontaneous mutation rate: Is it a health risk? Proceedings of the National Academy of Science 94, no. 23:8380-8386.

Diroma, M.A., C. Calabrese, D. Simone, M. Santorsola, F.M. Calabrese, G. Gasparre, and M. Attimonelli. 2014. Extraction and annotation of human mitochondrial genomes from 1000 Genomes Whole Exome Sequencing data. BMC Genomics 15(Suppl 3):S2.

Duret, L., and N. Galtier. 2009. Biased gene conversion and the evolution of mammalian genomic landscapes. Annual Reviews of Genomics and Human Genetics 10:285-311.

Elhaic, E., T. Tatarinova, A.A. Klyosov, and D. Graur. 2014. The 'extremely ancient' chromosome that isn't: a forensic bioinformatic investigation of Albert Perry's X-degenerate portion of the Y chromosome. European Journal of Human Genetics 22, no. 9:1111-1116.

Forster, L.P., Forster, S. Lutz-Bonengel, H. Willkomm, and B. Brinkmann. 2007. Natural radiation and human mitochondrial DNA mutations. Proceedings of the National Academy of Science 99, no. 21:1395013954.

Francioli, L.C., P.P. Polak, A. Koren, A. Menelaou, S. Chun, I. Renkens, Genome of the Netherlands Consortium, C.M. van Duijn, M. Swertz, C. Wijmenga, G. van Ommen, P. E. Slagboom, D.I. Boomsma, K. Ye, V. Guryev, P.F Arndt, W.P. Kloosterman, P.I.W de Bakker, and S.R. Sunyaev. 2015. Genome-wide patterns and properties of de novo mutations in humans. Nature Genetics 47, no. 7:822-826.

Groussin, M., J.K. Hobbs, G.J. Szöllösi, S. Gribaldo, V.L. Arcus, and M. Gouy. 2015. Toward more accurate ancestral protein genotypephenotype reconstructions with the use of species tree-aware gene trees Molecular Biology and Evolution 32, no. 1:13-22, 2015.

Haber, M., C. Doumet-Serhal, C. Scheib, Y. Xue, P. Danecek, M. Mezzavilla, S. Youhanna, R. Martiniano, J. Prado-Martinez, M. Szpak, E. Matisoo-Smith, H. Schutkowski, R. Mikulski, P. Zalloua, T. Kivisild, and C. Tyler-Smith. 2017. Continuity and admixture in the last five millennia of Levantine history from ancient Canaanite and presentday Lebanese genome sequences. The American Journal of Human Genetics 101:1-9.

Halldorsson, B.J., M.T. Hardarson, B. Kehr, U. Styrkarsdottir, A. Gylfason, G. Thorleifsson, F. Zink, A. Jonasdottir, A. Jonasdottir, P. Sulem, G. Masson, U. Thorsteinsdottir, A. Helgason, A. Kong, D.F. Gudbjartsson, and K. Stefansson. 2016. The rate of meiotic gene conversion varies by sex and age. Nature Genetics 48, no. 11:1377-1384.

Hallast, P., C. Batini, D. Zadik, P.M. Delser, J.H. Wetton, E. Arroyo-Pardo,
G.L. Cavalleri, P. de Knijff, G.D. Bisol, B.M. Dupuy, H.A. Eriksen, L.B. Jorde, T.E. King, M.H. Larmuseau, A.L. de Munain, A.M. LópezParra, A. Loutradis, J. Milasin, A. Novelletto, H. Pamjav, A. Sajantila, W. Schempp, M. Sears, A. Tolun, C. Tyler-Smith, A. Van Geystelen, S. Watkins, Bruce Winney, and M.A. Joblin. 2015. The Y-chromosome tree bursts into leaf: 13,000 high-confidence SNPs covering the majority of known clades. Molecular Biology and Evolution 32, no. 3:661-673.

Handt, O., S. Meyer, and A. von Haeseler. 1998. Compilation of human mtDNA control region sequences. Nucleic Acids Research 26, no. $1: 126-129$.

Hanson-Smith, V., B. Kolaczkowski, and J.W. Thornton. 2010. Robustness of ancestral sequence reconstruction to phylogenetic uncertainty. Molecular Biology and Evolution 27, no. 9:1988-1999.

Hardy, C., and R. Carter. 2014. The biblical minimum and maximum age of the earth. Journal of Creation 28, no. 2: 89-96.

Helgason, A., A.W. Einarsson, V.B. Guðmundsdóttir, Á. Sigurðsson, E.D. Gunnarsdóttir, A. Jagadeesan, S.S. Ebenesersdóttir, A. Kong, and K. Stefánsson. 2014. The Y-chromosome point mutation rate in humans. Nature Genetics 47, no. 5:453-457.

Henn, B.M., L.R. Botigué, S. Peischl, I. Dupanloup, M. Lipatov, B.K. Maples, A.R. Martin, S. Musharoff, H. Cann, M.P. Snyder, L. Excoffier, J.M. Kiddg, and C.D. Bustamante. 2015. Distance from sub-Saharan Africa predicts mutational load in diverse human genomes. Proceedings of the National Academy of Science 113, no. 4:E440-449.

Hinch, A.G., A. Tandon, N. Patterson, Y. Song, N. Rohland, C.D. Palmer, G.K. Chen, K. Wang, S.G. Buxbaum, E.L. Akylbekova, M.C. Aldrich, C.B. Ambrosone, C. Amos, E.V. Bandera, S.I. Berndt, L. Bernstein, W.J. Blot, C.H. Bock, E. Boerwinkle, Q. Cai, N. Caporaso, G. Casey, L.A. Cupples, S.L. Deming, W.R. Diver, J. Divers, M. Fornage, E.M. Gillanders, J. Glessner, C.C. Harris, J.J. Hu, S.A. Ingles, W. Isaacs, E.M. John, W.H.L. Kao, Brendan Keating, R.A. Kittles, L.N. Kolonel, E. Larkin, L. Le Marchand, L.H. McNeill, R.C. Millikan, A. Murphy, S. Musani, C.Neslund-Dudas, S. Nyante, G.J. Papanicolaou, M.F. Press, B.M. Psaty, A.P. Reiner, S. S. Rich, J.L. Rodriguez-Gil, J.I. Rotter, B.A. Rybicki, A.G. Schwartz, L.B. Signorello, M. Spitz, S.S. Strom, M.J. Thun, M.A. Tucker, Z. Wang, J.K. Wiencke, J.S. Witte, M. Wrensch, X. Wu, Y. Yamamura, K.A. Zanetti, W. Zheng, R.G. Ziegler, X. Zhu, S. Redline, J.N. Hirschhorn, B.E. Henderson, H.A. Taylor Jr., A.L. Price, H. Hakonarson, S.J. Chanock, C.A. Haiman, J.G. Wilson, D. Reich, and S.R. Myers. 2011. The landscape of recombination in African Americans. Nature 476:170-176.

Huelsenbeck, J.P., and J.P. Bollback. 2001. Empirical and hierarchical bayesian estimation of ancestral states. Systematic Biology 50, no. 3:351-366.

Jeanson, N.T. 2013. Recent, functionally diverse origin for mitochondrial genes from 2700 metazoan species. Answers Research Journal 6:467501.

Jeanson, N.T. 2015a. Mitochondrial DNA clocks imply linear speciation rates within "kinds." Answers Research Journal 8:273-304.

Jeanson, N.T. 2015b. A young-earth creation human mitochondrial DNA "clock": Whole mitochondrial genome mutation rate confirms D-loop results. Answers Research Journal 8:375-378.

Jeanson, N.T. 2016. On the origin of human mitochondrial DNA differences, new generation time data both suggest a unified youngearth creation model and challenge the evolutionary out-of-Africa model. Answers Research Journal 9:123-130.

Jermann, T.M., T.G. Opitz, J. Stackhouse, and S.A. Benner. 1995. Reconstructing the evolutionary history of the artiodactyl ribonuclease 
superfamily. Nature 374:57-59.

Johnson, M.J., D.C. Wallace, D.C. Ferris, M.C. Rattazzi, and L.L. CavalliSforza. 1983. Radiation of human mitochondria DNA types analyzed by restriction endonuclease cleavage patterns. Journal of Molecular Evolution 19, no. 3:255-271.

Karmin, M., L. Saag, M. Vicente, M.A.W Sayres, M. Järve, U.G. Talas. 2015. A recent bottleneck of $Y$ chromosome diversity coincides with a global change in culture. Genome Research 25:459-466.

Keenan, A., and A.G. Clark. 2012. Recent explosive human population growth has resulted in an excess of rare genetic variants. Science 336:740-743.

Kong, A., M.L. Frigge, G. Masson, S. Besenbacher, P. Sulem, G. Magnusson, S.A. Gudjonsson, A. Sigurdsson, A. Jonasdottir, A. Jonasdottir, W. Wong, G. Sigurdsson, G.B. Walters, S. Steinberg, H. Helgason, G. Thorleifsson, D.F. Gudbjartsson, A. Helgason, O.Th. Magnusson, U. Thorsteinsdottir, and K. Stefansson. 2012. Rate of de novo mutations, father's age, and disease risk. Nature 488, no. 7412:471-475.

Karafet, T.M., L. Mendez, M.B. Meilerman, P.A. Underhill, S.L. Zegura, and M.F. Hammer. 2008. New binary polymorphisms reshape and increase resolution of the human $\mathrm{Y}$ chromosomal haplogroup tree. Genome Research 18:8930-838.

Krašovec, R., H. Richards, D.R. Gifford, C. Hatcher, K.J. Faulkner, R.V. Belavkin, A. Channon, E. Aston, A.J. McBain, and C.G. Knight. 2017. Spontaneous mutation rate is a plastic trait associated with population density across domains of life. PLOS Biology 15(8):e2002731.

Langergraber, K.E., 2009. Generation times in wild chimpanzees and gorillas suggest earlier divergence times in great ape and human evolution. Proceedings of the National Academy of Science 109, no. 39:15716-15721.

Lesecque, Y., D. Mouchiroud, and L. Duret. 2013. GC-biased gene conversion in yeast is specifically associated with crossovers: molecular mechanisms and evolutionary significance. Molecular Biology and Evolution 30, no. 6:1409-1419.

Loogväli, E.L., T. Kivisild, T. Margus, and R. Villems. 2009. Explaining the imperfection of the molecular clock of hominid mitochondria. PLoS One 4, no. 12: e8260.

Lopez, P., D. Casane, and H. Philippe. 2002. Heterotachy, an important process of protein evolution. Molecular Biology and Evolution 19, no. $1: 1-7$.

Madrigal, L., L. Castrì, M. Melendez-Obando, R.Villegas-Palma, R. Barrantes, H. Raventos, R. Pereira, D. Luiselli, D. Pettener, and G. Barbujani. 2012. High mitochondrial mutation rates estimated from deep-rooting Costa Rican pedigrees. American Journal of Physical Anthropology 148:327-333.

Mallik, S., H. Li, M. Lipson, I. Mathieson, M. Gymrek, F. Racimo, 2016. The Simons Genome Diversity Project: 300 genomes from 142 diverse populations. Nature 538:201-206.

Mendez, F.L., T. Krahn, B. Schrack, A-.M. Krahn, K.R. Veeramah, A.E. Woerner, F.L.M. Fomine, N. Bradman, M.G. Thomas, T.M. Karafet, and M.F. Hammer. 2013. An African American paternal lineage adds an extremely ancient root to the human Y chromosome phylogenetic tree. The American Journal of Human Genetics 92:454-459.

Moorjani, P., C.E.G Amorim, P.F. Arndt, and M. Przeworski. 2016. Variation in the molecular clock of primates. Proceedings of the National Academy of Science 113, no. 38:10607-10612.

Pauling L., and E. Zuckerkandl. 1963. Chemical paleogenetics, molecular "restoration studies" of extinct forms of life. Acta Chemica Scandinavica 17, no 89: 9-16.

Pinto, C., M. Pinheiro, A. Peixoto, C. Santos, I. Veiga, P. Rocha, P. Pinto, P. Lopes, M. Baptista, R. Henrique, and M.R. Teixeira. 2016. Co-occurrence of nonsense mutations in MSH6 and MSH2 in Lynch syndrome families evidencing that not all truncating mutations are equal. Journal of Human Genetics 61, no. 2:151-6.

Poznik, G.D., B.M. Henn, M.-C.Yee, E. Sliwerska, G.M. Euskirchen, A.A. Lin, M. Snyder, L. Quintana-Murci, J.M. Kidd, P.A. Underhill, and C.D. Bustamante. 2013. Sequencing Y chromosomes resolves discrepancy in time to common ancestor of males versus females. Science 341:562 565 .

Poznik, G.D., Y. Xue, F.L. Mendez, T.F. Willems, A. Massaia, M.A. Wilson Sayres, Q. Ayub, S.A. McCarthy, A. Narechania, S. Kashin, Y. Chen, R. Banerjee, J.L. Rodriguez-Flores, M. Cerezo, H. Shao, M. Gymrek, A. Malhotra, S. Louzada, R. Desalle, G.R.S. Ritchie, E. Cerveira, T.W. Fitzgerald, E. Garrison, A. Marcketta, D. Mittelman, M. Romanovitch, C. Zhang, X. Zheng-Bradley, G.R. Abecasis, S.A. McCarroll, P. Flicek, P.A. Underhill, L. Coin, D.R. Zerbino, F. Yang, C. Lee, L. Clarke, A. Auton, Y. Erlich, R.E. Handsaker, The 1000 Genomes Project Consortium, C.D. Bustamante, and C. Tyler-Smith. 2016. Punctuated bursts in human male demography inferred from 1,244 worldwide Y-chromosome sequences. Nature Genetics 48:593-599.

Pupko, T., I.P.R. Shamir, and G. Graur. 2000. A fast algorithm for joint reconstruction of ancestral amino acid sequences. Molecular Biology and Evolution 17, no. 6:890-896.

Purps, J., S. Siegert, S. Willuweit, M. Nagy, C. Alves, R. Salazar, et al. 2014. A global analysis of Y-chromosomal haplotype diversity for 23 STR loci. FSI:Genetics 12:12-23.

Rahbari, R., A. Wuster, S.J. Lindsay, R.J. Hardwick, L.B. Alexandrov, S.A. Turki, A. Dominiczak, A. Morris, D. Porteous, B. Smith, M.R. Stratton, UK10K Consortium, and M.E. Hurles. 2015. Timing, rates and spectra of human germline mutation. Nature Genetics 48, no. 2:126-133.

Reich, D., R.E. Green, M. Kircher, J. Krause, N. Patterson, E.Y. Durand B. Viola, A.W. Briggs, U. Stenzel, P.L.F. Johnson, T. Maricic, J.M. Good, T. Marques-Bonet, C. Alkan, Q. Fu, S. Mallick, H. Li, M. Meyer, E.E. Eichler, M. Stoneking, M. Richards, S. Talamo, M.V. Shunkov, A.P. Derevianko, J.-J. Hublin, J. Kelso, M. Slatkin, and S. Pääbo. 2010. Genetic history of an archaic hominin group from Denisova Cave in Siberia. Nature 468:1053-1060.

Romualdi, C., D. Balding, I.S. Nasidze, G. Risch, M. Robichaux, S.T. Sherry, M. Stoneking, M.A. Batzer, and G. Barbujani. 2002. Patterns of human diversity, within and among continents, inferred from biallelic DNA polymorphisms. Genome Research 12:602-612.

Rozen, S., H. Skaletsky, J.D. Marszalek, P.J. Minx, H.S. Cordum, R.H. Waterston, R.K. Wilson, and D.C. Page. 2003. Abundant gene conversion between arms of palindromes in human and ape Y chromosomes. Nature 423:873-876.

Rupe, C.L., and J.C. Sanford. 2013. Using numerical simulation to better understand fixation rates, and establishment of a new principle: Haldane's Ratchet) Proceedings of the Seventh International Conference on Creationism. In ed. M.Horstemeyer, Pittsburgh, Pennsylvania: Creation Science Fellowship.

Sayres, M.A.W., K.E. Lohmueller, and R. Nielsen. 2014. Natural selection reduced diversity on human Y chromosomes. PLoS Genetics 10, no. 1:e1004064.

Schuenemann, V.J., A. Peltzer, B. Welte, W.P. van Pelt, M. Molak, C.-C. Wang, A. Furtwängler, C. Urban, E. Reiter, K. Nieselt, B. TeBmann, 
M. Francken, K. Harvati, W. Haak, S. Schiffels, and J. Krause. 2016. Ancient Egyptian mummy genomes suggest an increase of Sub-Saharan African ancestry in post-Roman periods. Nature Communications 8:15694.

Schlebusch, C.M., H. Malström, T. Günther, P. Sjödin, A. Coutinho, H. Edlund, A.R. Munters, M. Vicente, M. Steyn, H. Soodyall, M. Lombard, and M. Jakobsson. 2017. Southern African ancient genomes estimate modern human divergence to 350,000 to 260,000 years ago. Science 358, no. 6363:652-655.

Scozzari, R., A. Massaia, B. Trombetta, G. Bellusci, N.M. Myres, A. Novelletto, and F. Cruciani. 2014. An unbiased resource of novel SNP markers provides a new chronology for the human Y chromosome and reveals a deep phylogenetic structure in Africa. Genome Research 24:535-544.

Sigurðaróttir, S., A. Helgason, J.R. Gulcher, K. Stefansson, and P. Donnelly. 2000. The mutation rate in the human mtDNA control region. American Journal of Human Genetics 66:1599-1609.

Skogland P., J.C. Thompson, M.E. Prendergast, A. Mittnik, K. Sirak, M. Hajdinjak, T. Salie, N. Rohland, S. Mallick, A. Peltzer, A. Heinze, I. Olalde, M. Ferry, E. Harney, M. Michel, K. Stewardson, J.I. CerezoRomán, C. Chiumia, A. Crowther, E. Gomani-Chindebvu, A.O. Gidna, K.M. Grillo, I.T. Helenius, G. Hellenthal, R. Helm, M. Horton, S. López, A.Z. Mabulla, J. Parkington, C. Shipton, M.G. Thomas, R. Tibesasa, M. Welling, V.M. Hayes, D.J. Kennett, R. Ramesar, M. Meyer, S. Pääbo, N. Patterson, A.G. Morris, N. Boivin, R. Pinhasi, J. Krause, and D. Reich. 2017. Reconstructing prehistoric African population structure. Cell 171, no. 1: 59-71.

Skov, L., The Danish Pan Genome Consortium [ $2^{\text {nd }}$ author], and M. H Schierup. 2017. Analysis of 62 hybrid assembled human Y chromosomes exposes rapid structural changes and high rates of gene conversion. PLOS Genetics 13, no. 8:e1006834.

Slatkin, M., and F. Racimo. 2016. Ancient DNA and human history. Proceedings of the National Academy of Science 113, no. 23:6380-6387.

Soares, P., L. Ermini, N. Thomson, M. Mormina, T. Rito, A. Röhl, A. Salas, S. Oppenheimer, V. Macaulay, and M.B. Richards. 2009. Correcting for purifying selection: an improved human mitochondrial molecular clock. American Journal of Human Genetics 84:740-759.

Smith, D.R. 2015. The past, present and future of mitochondrial genomics: have we sequenced enough mtDNAs? Briefings in Functional Genomics doi:10.1093/bfgp/elv027.

Tamura, K., G. Stecher, D. Peterson, A. Filipski, and S. Kumar. 2013. MEGA6: Molecular evolutionary genetics analysis version 6.0. Molecular Biology and Evolution 30:2725-2729.

Tenaillon, O., J.E. Barrick, N. Ribeck, D.E. Deatherage, J.L. Blanchard, A. Dasgupta, G.C. Wu, S. Wielgoss, S. Cruveiller, C. Médigue, D. Schneider, and R.E. Lenski. 2016. Tempo and mode of genome evolution in a 50,000-generation experiment. Nature 536, no. 7615:165-170.

Trombetta, B., D. Sellitto, R. Scozzari, and F. Cruciani. 2014. Interand intraspecies phylogenetic analyses reveal extensive $\mathrm{X}-\mathrm{Y}$ gene conversion in the evolution of gametologous sequences of human sex chromosomes. Molecular Biology and Evolution 31, no. 8: 2108-2123.

Thomas, B., and J. Tompkins. 2014. How reliable are genomes from ancient DNA? Journal of Creation 28, no.3:92-98.

Tomkins, J.P. 2015. Empirical genetic clocks give biblical timelines, Journal of Creation 29, no. 2:3-5, 2015.

Tompkins, J.P., and J. Bergman. 2015. Evolutionary molecular genetic clocks - a perpetual exercise in futility and failure, Journal of Creation 29, no. 2:26-35.

Trombetta, B., and F. Cruciani. 2017. Y chromosome palindromes and gene conversion. Human Genetics 136, no. 5:605-619.

Wood, T.C. 2012. Ancient mtDNA implies a nonconstant molecular clock in the human holobaramin. Journal of Creation Theology and Science Series B: Life Sciences 2:18-26.

Wood, T.C. 2013. Mitochondrial DNA analysis of three terrestrial mammal baramins (Equidae, Felidae, and Canidae) implies and accelerated mutation rate near the time of the Flood. In Proceedings of the Seventh International Conference on Creationism, ed. M. Horstemeyer, Pittsburgh, Pennsylvania: Creation Science Fellowship

Yang, S., L. Wang, J. Huang, X. Zhang, Y. Yuan, J-.Q. Chen, L.D. Hurst, and D. Tian. 2015. Parent-progeny sequencing indicates higher mutation rates in heterozygotes. Nature 523:463-467.

Yang, M.A., and Q. Fu. 2018. Insights into modern human prehistory using ancient genomes. Cell 34, no 3: 184-196.

\section{THE AUTHORS}

Robert Carter received his $\mathrm{PhD}$ from the University of Miami in 2003. With a background in genetics, gene cloning and sequencing, and transgenics, he soon began to work on the GENE project team (ICR), then as a speaker for Creation Ministries International. Rob was the lead editor of Evolution's Achilles' Heels (the book) and a co-producer and codirector of the award-winning documentary by the same name. Over the years, he has published multiple papers directed toward building a biblical model of human genetic history.

Dr. Stephen Sauchi Lee is a Professor of Statistics and an Affiliate Professor of Bioinformatics and Computational Biology in the Department of Statistical Science at the University of Idaho, Moscow, ID, USA. Dr. Lee's research interests are in Data Science, Statistical/Machine Learning, Multivariate Analysis, Computational Methods, Bioinformatics/ Genomics, Text Mining, and Visualization. He teaches a wide range of undergraduate and graduate level statistics courses.

As a Cornell University professor, John Sanford has been conducting genetic research for over 30 years. This research has resulted in more than 100 scientific publications, and several dozen patents. John is presently a Courtesy Associate Professor at Cornell, President of Logos Research Associates, and President of Feed My Sheep Foundation. John's most significant contributions to science have been: 1) the Biolistic Process; 2) the book Genetic Entropy; 3) Development of Mendel's Accountant (a comprehensive and biologically realistic numerical simulation of the mutation/selection process); and 4) lead organizer/editor of the Cornell symposium and subsequently published proceedings entitled Biological Information--New Perspectives. 